\title{
PriSTE: From Location Privacy to Spatiotemporal Event Privacy
}

\author{
Yang $\mathrm{Cao}^{* \uparrow \|}$, Yonghui Xiao ${ }^{\ddagger * *}$, Li Xiong ${ }^{\dagger}$, Liquan $\mathrm{Bai}^{\S \uparrow}$ \\ Emory University, Atlanta, U.S.A \\ "Kyoto University, Kyoto, Japan \\ ${ }^{* *}$ Google Inc., Mountain View, U.S.A \\ Email: *yang@i.kyoto-u.ac.jp †lxiong@emory.edu, †yhandxiao@gmail.com, §bailiquan@gmail.com
}

\begin{abstract}
Location privacy-preserving mechanisms (LPPMs) have been extensively studied for protecting a user's location at each time point or a sequence of locations with different timestamps (i.e., a trajectory). We argue that existing LPPMs are not capable of protecting the sensitive information in user's spatiotemporal activities, such as "visited hospital in the last week" or "regularly commuting between Address 1 and Address $\mathbf{2}$ every morning and afternoon" (it is easy to infer that Addresses 1 and 2 may be home and office). We define such privacy as Spatiotemporal Event Privacy, which can be formalized as Boolean expressions between location and time predicates. To understand how much spatiotemporal event privacy that existing LPPMs can provide, we first formally define spatiotemporal event privacy by extending the notion of differential privacy, and then provide a framework for calculating the spatiotemporal event privacy loss of a given LPPM under attackers who have knowledge of user's mobility pattern. We also show a case study of utilizing our framework to convert the state-of-the-art mechanism for location privacy, i.e., Planner Laplace Mechanism for Geo-indistinguishability, into one protecting spatiotemporal event privacy. Our experiments on real-life and synthetic data verified that the proposed method is effective and efficient.
\end{abstract}

\section{INTRODUCTION}

The continued advances and usage of smartphones and GPS-enabled devices have provided tremendous opportunities for Location-Based Service (LBS), such as Google Maps, Facebook Places and Swarm. In the location-based services, mobile users have to share their locations or trajectories with the service providers in order to issue snapshot or continuous queries, for example, "where is the nearest gas station" or "continuously report the taxis within one mile of my location". It has raised privacy concerns as users' digital trace can be used to infer sensitive information, such as home and work place, religious places and sexual inclinations [1] [2] [3].

A large number of studies (see surveys [4] [5] [6] [7]) have explored how to protect user's location privacy from different aspects: privacy goals, adversarial models, location privacy metrics, and Location Privacy Preserving Mechanisms (LPPMs). Privacy goals indicate what should be protected or what are the secrets (e.g., a single location or a trajectory); adversarial models make assumptions about the adversaries; location privacy metrics formally define the quantitative method for the privacy goal (e.g., Geo-indistinguishability [8]

Yang and Yonghui contributed equally to this work. or $\delta$-location set privacy [9]); LPPMs study how to achieve a specified privacy metric.

We argue that existing LPPMs may not be able to fully protect users' sensitive information in their spatiotemporal activities because their privacy goal is focused on protection of either a single location or a trajectory. A user's location data can be represented by tuple (we consider the single user setting in this paper), i.e., <location, time>, which corresponds to information about "where" and "when" in user's real-world activities. Hence, the privacy goals in literature can be categorized into protecting a single position or a trajectory. Many LPPMs are proposed for these goals based on different privacy metrics. For example, Gruteser et al. [10] designed a spatiotemporal cloaking mechanism satisfying $\mathrm{k}$ anonymity to protect movement trajectories of users; Andrés et al. [8] proposed Planar Laplace mechanism [8] achieving Geo-indistinguishability to protect single locations; Xiao and Xiong [9] designed Planar Isotropic Mechanism for $\delta$-location set privacy to protect each location in a trajectory.

However, the privacy goals in the literature of location privacy only attempt to confuse adversaries about either the user's exact location or trajectory, and the two types of privacy goals cannot cover all cases of complex combination of spatial and temporal information (as shown in Fig 1), which we refer to as spatiotemporal events in this paper. Examples of spatiotemporal event include "visited hospital in the last week" (i.e., the hospital visit may happen once or multiple times at any time in last week) and "regularly commuting between Address 1 and Address 2 every morning and every afternoon" (these periodic spatiotemporal events may happen every day).

We show six cases of the Boolean expression between location and time predicates in Fig 1. It turns out that protecting a single location or a trajectory are only two cases among possible privacy goals in protecting a user's spatial and temporal information. Let $u^{t}$ be a user's position at time $t$, and $s_{i} \in \mathbb{S}, i \in[1, m]$ be one of all $m$ locations on the map. As shown in Fig.1, the element of a user's secrets in her spatiotemporal activities can be represented by a predicate $u^{t}=s_{i}$ (the value can be either true or false). Then, a spatiotemporal event can be defined as a Boolean expression by combining different predicates over spatial and/or temporal dimensions (a predicate alone also can be a spatiotemporal 
event). As shown in Fig, 1, the events representing a sensitive location/area and a trajectory, which are the main focuses in previous studies of location privacy, are only two cases (i.e., (b) and (c)) in the six enumerated examples. Even if each location or a trajectory is protected, it is not clear whether or not adversary is able to infer the value of a sensitive spatiotemporal event. Protecting the privacy of spatiotemporal events has not been studied in literature.

\begin{tabular}{|c|c|c|c|c|c|}
\hline \multicolumn{3}{|c|}{ Spatial dimension } & \multicolumn{2}{|c|}{ Temporal dimension } & Spatial and Temporal \\
\hline & $u^{1}$ & $u^{2}$ & & $u^{1}$ & $u^{1}$ \\
\hline$s_{1}$ & P & $\circ$ & $s_{1}$ & OAND-O & 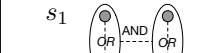 \\
\hline$s_{2}$ & 0 & 0 & $s_{2}$ & 0 & $\left.\begin{array}{lll}s_{2} & 0 & 0\end{array}\right)$ \\
\hline \multicolumn{3}{|c|}{$(a)\left(u^{1}=s_{1}\right) \wedge\left(u^{1}=s_{2}\right)$} & \multicolumn{2}{|c|}{$(c)\left(u^{1}=s_{1}\right) \wedge\left(u^{2}=s_{1}\right)$} & $\begin{aligned} &(e)\left(\left(u^{1}=s_{1}\right) \vee\left(u^{1}=s_{2}\right)\right) \\
& \wedge\left(\left(u^{2}=s_{1}\right) \vee\left(u^{2}=s_{2}\right)\right)\end{aligned}$ \\
\hline & $u^{1}$ & $u^{2}$ & & $u^{1}$ & $u^{2}$ \\
\hline$s_{1}$ & $\stackrel{p}{d R}$ & $\circ$ & $s_{1}$ & $O O R \ldots$ & 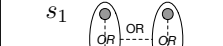 \\
\hline$s_{2}$ & 0 & 0 & $s_{2}$ & $\circ$ & $\begin{array}{lll}s_{2} & 0\end{array}$ \\
\hline \multicolumn{3}{|c|}{ (b) $\left(u^{1}=s_{1}\right) \vee\left(u^{1}=s_{2}\right)$} & \multicolumn{2}{|c|}{$(d)\left(u^{1}=s_{1}\right) \vee\left(u^{2}=s_{1}\right)$} & $\begin{aligned}(f) & \left(\left(u^{1}=s_{1}\right) \vee\left(u^{1}=s_{2}\right)\right) \\
\vee & \left(\left(u^{2}=s_{1}\right) \vee\left(u^{2}=s_{2}\right)\right)\end{aligned}$ \\
\hline
\end{tabular}

Fig. 1: Examples of spatiotemporal events. $s_{1}$ and $s_{2}$ are two locations on the map $\mathbb{S} . u^{1}$ and $u^{2}$ are two variables about user's possible locations at time point 1 and time point 2, respectively. Event (a) is always false since a user cannot be at two different locations at the same time. Event (b) means that the secret is a sensitive area including locations $\left\{s_{1}, s_{2}\right\}$. Event (c) represents a sensitive trajectory $s_{1} \rightarrow s_{1}$. Event (d) denotes that the secret is the visit to $s_{1}$ at time point 1 or 2 . Event (e) depicts the secret as a type of trajectory pattern, i.e., the user may stay at two sensitive areas successively. Event (f) indicates the secret as user's presence in sensitive area $\left\{s_{1}, s_{2}\right\}$ at either time point 1 or 2 .

Although an LPPM protecting a single location or a trajectory (i.e., Fig 1 (b) or Fig 1 (c)) ensures user's location privacy, it is not clear whether such mechanisms also provide a certain level of spatiotemporal event privacy (such as Fig,1 (e) or Fig 1(f)). In this paper, we attempt to formalize the new privacy goal of spatiotemporal event privacy and quantify how much spatiotemporal event privacy that an existing LPPM can provide. Towards this goal, in addition to the lack of definition and privacy metrics for spatiotemporal event, a challenge is the computational complexity for quantifying the privacy loss of a given LPPM w.r.t a specified spatiotemporal event. For example, given a complex spatiotemporal event, i.e., a Boolean expression, checking its value (true or false) requires enumeration of all possible values of the predicates in the Boolean expression, which can be exponential to the number of predicates.

Contributions. In this paper, we study how to protect spatiotemporal event privacy for the first time. Our contributions are summarized as follows.

First, we investigate the privacy goal and privacy metric for spatiotemporal event privacy. We formally define the new type of privacy goal, i.e., spatiotemporal events, as Boolean expressions of a set of (location, time) predicates, and propose a privacy metric for protecting spatiotemporal events by extending the notion of differential privacy which we call $\epsilon$ - spatiotemporal event privacy. We also explore the difference between the metrics of location privacy and spatiotemporal event privacy. It turns out that, although the privacy goal of spatiotemporal event privacy is more general than location privacy, the privacy metrics can be orthogonal. Hence, it would be preferable that an LPPM achieving a location privacy metric such as Geo-indistinguishability can also satisfy $\epsilon$ spatiotemporal event privacy w.r.t. user-specified events. Location privacy provides general protection against unknown risks, while spatiotemporal event privacy guarantees flexible and customizable protection which may not be provided by the existing LPPMs.

Second, we develop a method for quantifying how much $\epsilon$-spatiotemporal event privacy a given LPPM can provide. In this paper, we model an LPPM as an emission matrix that takes user's true position as input and outputs a perturbed location. As we mentioned previously, one of the challenges in quantifying the probability of a spatiotemporal event is that the computational complexity may be exponentially increasing with the number of its predicates. We develop a two-possibleworld method to quantify spatiotemporal event privacy with linear complexity to the number of predicates.

Third, based on our quantification method, we propose

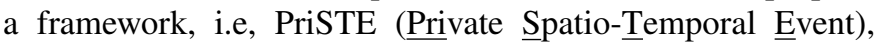
which converts a mechanism for location privacy into one for spatiotemporal event privacy. We demonstrate the effectiveness of our framework using two case studies using Geoindistinguishability [8] and $\delta$-location set privacy [9], which are the state-of-the-art location privacy metrics.

Finally, we implement and evaluate our algorithms on both synthetic and real-world datasets evaluating its feasibility, efficiency, and the impact of various parameters.

\section{Problem Setting AND Definitions}

\section{A. Problem Setting}

We study how to protect spatiotemporal event in a single user setting. Consider a user who is sharing her location sequence with a location-based service provider. Since a system with a trusted server is vulnerable to single point of attack, the user does not want to share her sensitive information with the server; instead, she uses a local LPPM that guarantees location privacy at each timestamp. We denote a moving user's true locations as $\left\{u^{1}, u^{2}, \cdots, u^{T}\right\}$. The LPPM blurs user's true location $u^{t}$ to a perturbed one $o_{t}$ that satisfies a privacy metric such as geo-indistinguishability [8] or $\delta$-location set privacy [9]. Hence, the LPPM can be considered as an emission matrix that takes user's true location as input and outputs a perturbed one. The major notations in this paper are summarized in Table [1]

\section{B. Spatiotemporal Events}

Spatiotemporal events can represent user's secrets in their real-world activities, such as "visited hospital in the last week" or "commuting between Address 1 and Address 2 every morning and afternoon". Let $\mathbb{S}=\left\{s_{1}, s_{2}, \cdots, s_{m}\right\}$ be the domain of space, where $m$ is the number of all locations and 


\begin{tabular}{|l|l|}
\hline $\mathbf{s}$ & a region consists of some states, $\mathbf{s} \in\{0,1\}^{m \times 1}$ \\
\hline$t$ & one timestamp in $\{1,2, \cdots, T\}$ \\
\hline $\mathbf{S}$ & a set of regions $\mathbf{s}$ \\
\hline $\mathbf{T}$ & a set of timestamps \\
\hline$u^{t}$ & a user's true location at time $t$ \\
\hline$o^{t}$ & a user's perturbed location at time $t$ \\
\hline EVENT & a spatiotemporal event in time $\{$ start, $\cdots$, end $\}$ \\
\hline$\tilde{\mathbf{p}}_{o_{t}}$ & a vector of emission probabilities given the observation $o_{t}$. \\
\hline$\tilde{\mathbf{p}}_{o_{t}}^{\mathbf{D}}$ & a diagonal matrix with the vector $\tilde{\mathbf{p}}_{o_{t}}$ on the diagonal. \\
\hline
\end{tabular}

TABLE I: Notations

$s_{i}$ is one location (we use state interchangeably) on the map. A user's trajectory consists of a set of $\{u, t\}$ denoting the user's location at timestamp $t$ in $\{1,2, \cdots, T\}$. Each pair of location and time can be represented by a predicate. For example, the pair $\left\{u^{1}, s_{3}\right\}$ can be denoted by a predicate $u^{1}=s_{3}$. If the user is in location $s_{3}$ at timestamp 1, then the ground truth of the predicate is true. A spatiotemporal event is defined as a Boolean expression of the (location, time) predicates using the AND, OR, NOT operators, denoted by $\wedge, \vee, \neg$ respectively. Definition II.1 (EVENT). A spatiotemporal event, denoted by EVENT, is a set of (location, time) predicates, i.e. $u^{t}=s_{i}$, under the Boolean operations.

Using Boolean logic to define spatiotemporal events enable users to customize their privacy preference in diverse realworld activities. Table II shows some representative examples of EVENT. If a user is in a state $s_{i}$ at timestamp $t$, then $u^{t}=s_{i}$. If the user is in a region of $\left\{s_{i}, s_{j}, \cdots, s_{k}\right\}$ at timestamp $t$, then $\left(u^{t}=s_{i}\right) \vee\left(u^{t}=s_{j}\right) \vee \cdots, \vee\left(u^{t}=s_{k}\right)$ holds. If the trajectory of the user is $\left\{s_{i}, s_{j}, \cdots, s_{k}\right\}$ over timestamps 1 to $T$, then $\left(u^{1}=s_{i}\right) \wedge\left(u^{2}=s_{j}\right) \wedge \cdots, \wedge\left(u^{t}=s_{k}\right)$ holds. Based on the Boolean operations, complicated spatiotemporal events can be defined as follows.

PresenCE. When the secret is whether or not a user visited a sensitive area (e.g., medical facilities) in a given time period, we can use PRESENCE to represent such secret. A PRESENCE event holds if a user appears in a region during some time. In the simplest case, the region consists of one location, and time period consists of one timestamp, then it becomes one single location shown in Table II Hence, PRESENCE is a generalization of secrets about single locations. To denote a region, which is a set of locations, we use a vector $\mathbf{s} \in\{0,1\}^{m \times 1}$ where the $i$ th element is 1 if the region contains $s_{i}$. The time period is denoted by $\mathbf{T}$ as a set of timestamps.

Definition II.2 (PRESENCE). Given a set of regions $\boldsymbol{S}$ and $a$ time period $\boldsymbol{T}$, if a user appears in $\boldsymbol{s}$ at any timestamp $t \in \boldsymbol{T}$, then it is a presence event, denoted by $\operatorname{PrEsence}(\boldsymbol{S}, \boldsymbol{T})$.

Example II.1 (PRESENCE). Fig 2 shows a map of $\mathbb{S}=$ $\left\{s_{1}, s_{2}, s_{3}\right\}$. The shaded region shows a PATTERN event that the user appears in a region of $s_{1}$ or $s_{2}$ during timestamps 3 and 4 . The lines indicate possible trajectories. As long as user's true trajectory passes through the shaded region, the ground truth of the event is true. For this event, the region $\boldsymbol{s}=[1,1,0]^{\top}$ denoting the states $s_{1}$ and $s_{2}$; the time period $\boldsymbol{T}=\{3,4\}$ denoting timestamp 3 and 4 . The PRESENCE event is expressed as $\left(u^{3}=s_{1}\right) \vee\left(u^{3}=s_{2}\right) \vee\left(u^{4}=s_{1}\right) \vee\left(u^{4}=s_{2}\right)$. PatTern. When the secret is whether or not a user visited

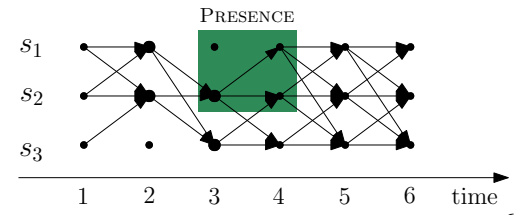

Fig. 2: PRESENCE: the user appears in regions of $\left\{s_{1}, s_{2}\right\}$ during timestamps 3 and 4; PATTERN: the trajectories all go through $\left\{s_{1}, s_{2}\right\}$ at timestamp 2 and $\left\{s_{2}, s_{3}\right\}$ at timestamp 3 .

multiple sensitive areas successively (e.g., a love hotel and then home), we can use PRESENCE to represent such secret. In a simple case, the regions consist of single locations at a set of timestamps, then it becomes single trajectory shown in Table III. Hence, PATTERN is a generalization of secrets about user's trajectories. We define PATTERN as follows where the set of regions $\left[\mathbf{s}_{1}, \mathbf{s}_{2}, \cdots, \mathbf{s}_{n}\right]$ is denoted by $\mathbf{S}$.

Definition II.3 (PATTERN). Given a sequence of regions $\boldsymbol{S}=$ $\left\{\boldsymbol{s}_{1}, \boldsymbol{s}_{2}, \cdots, \boldsymbol{s}_{n}\right\}$ and a time period $\boldsymbol{T}$ where $\boldsymbol{s}_{i} \in\{0,1\}^{m \times 1}$, if a user appears in $\left\{\boldsymbol{s}_{1}, \boldsymbol{s}_{2}, \cdots, \boldsymbol{s}_{n}\right\}$ sequentially during $\boldsymbol{T}$, then it is a pattern event, denoted by $\operatorname{PATTERN}(\boldsymbol{S}, \boldsymbol{T})$.

Example II.2 (PATTERN). Fig2 2 shows a set of trajectories with a PATTERN that all trajectories go through $\left\{s_{1}, s_{2}\right\}$ at timestamp 2 and $\left\{s_{2}, s_{3}\right\}$ at timestamp 3. For this event, the region at timestamp 2 is $\boldsymbol{s}_{2}=[1,1,0]^{\top}$ denoting $s_{1}$ and $2_{2}$; the region at timestamp 3 is $s_{3}=[0,1,1]^{\top}$ denoting $s_{2}$ and $s_{3}$. The PATTERN event is expressed as $\left(\left(u^{2}=s_{1}\right) \vee\left(u^{2}=s_{2}\right)\right) \wedge\left(\left(u^{3}=\right.\right.$ $\left.\left.s_{2}\right) \vee\left(u^{3}=s_{3}\right)\right)$.

From the above definitions, we can see that, in terms of privacy goal, spatiotemporal event privacy is a generalization of location privacy. In this paper, we focus on the two representative events defined above, i.e., Presence and Pattern, which are the two most complicated events in examples of Fig 1 We note that PRESENCE and PATTERN include the cases when the time $\mathbf{T}$ is not consecutive. For simplicity, we assume that the events are defined in consecutive time and use start and end to denote the start point and end point of the defined spatiotemporal event. Users can customize one or multiple spatiotemporal events to be protected.

Protecting spatiotemporal events requires a local mechanism that is able to localize the information about spatiotemporal event. We need a formal privacy metric to preserve user's plausible deniability about the truth of her specified spatiotemporal events, so that even when adversaries happen to infer the specified spatiotemporal event, the user has plausible deniability. We propose such a privacy metric for spatiotemporal event privacy in the next section.

\section{C. $\epsilon$-Spatiotemporal Event Privacy}

Inspired by the definition of differential privacy [11], we define $\epsilon$-Spatiotemporal Event Privacy as follows.

Definition II.4 ( $\epsilon$-Spatiotemporal Event Privacy). A mechanism preserves $\epsilon$-Spatiotemporal Event Privacy for a spatiotemporal EVENT if at any timestamp $t$ in $\{1,2, \cdots, T\}$ given any observations $\left\{o_{1}, o_{2}, \cdots, o_{T}\right\}$,

$$
\operatorname{Pr}\left(o_{1}, o_{2}, \cdots, o_{t} \mid \operatorname{EVENT}\right) \leq e^{\epsilon} \operatorname{Pr}\left(o_{1}, o_{2}, \cdots, o_{t} \mid \neg \text { EVENT }\right)
$$




\begin{tabular}{|c|c|c|}
\hline EVENT & Boolean Expression & Interpretation \\
\hline single location & $u^{t}=s_{i}$ & the location at timestamp $t$ is $s_{i}$ \\
\hline PRESENCE at $s_{i}$ during $T$ & $\left(u^{1}=s_{i}\right) \vee\left(u^{2}=s_{i}\right) \vee \cdots \vee\left(u^{t}=s_{i}\right)$ & appears at location $s_{i}$ during time $\{1,2, \cdots, T\}$ \\
\hline PRESENCE at $\mathbf{s}$ and $t$ & $\left(u^{t}=s_{i}\right) \vee\left(u^{t}=s_{j}\right) \vee \cdots, \vee\left(u^{t}=s_{k}\right)$ & appears in region $\mathbf{s}:\left\{s_{i}, \cdots, s_{k}\right\}$ at timestamp $t$ \\
\hline single trajectory & $\left(u^{1}=s_{i}\right) \wedge\left(u^{2}=s_{j}\right) \wedge \cdots, \wedge\left(u^{n}=s_{k}\right)$ & a trajectory of locations during a time period \\
\hline PATTERN of trajectories & $\left(\left(u^{1}=s_{i}\right) \vee\left(u^{1}=s_{j}\right) \vee \cdots, \vee\left(u^{1}=s_{k}\right) \wedge \cdots\right.$ & a PATTERN of trajectories \\
& $\wedge\left(\left(u^{n}=s_{l}\right) \vee\left(u^{n}=s_{m}\right) \vee \cdots,\left(u^{n}=s_{n}\right)\right)$ & \\
\hline
\end{tabular}

TABLE II: EVENTS of Boolean operations on the (location, time) predicates

$\begin{array}{llll}\text { (a) indistinguishability-based } & \text { (b) indistinguishability-based } & \text { (c) } \varepsilon \text {-Spatiotemporal Event Privacy }\end{array}$ Location Privacy

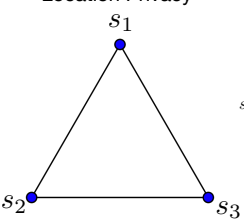
Trajectory Privacy
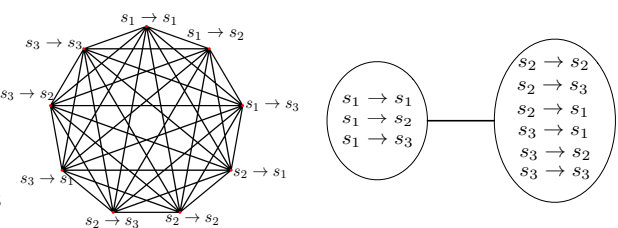

Fig. 3: Illustration of indistinguishability-based privacy metrics for distinct privacy goals when $\mathbb{S}=\left\{s_{1}, s_{2}, s_{3}\right\}$ and $T=2$.

where EVENT is a logic variable about the defined spatiotemporal event and $\neg$ EVENT denotes the negation of EVENT. $\operatorname{Pr}\left(o_{1}, o_{2}, \cdots, o_{t} \mid\right.$ EVENT $)$ denotes the probability of the observations $o_{1}, o_{2}, \cdots, o_{t}$ given the value of EVENT.

There are two major benefits of adopting such "DP-like" privacy metric. First, it provides a well-defined semantics for spatiotemporal event privacy. Similar to differential privacy that requires the indistinguishability between any two neighboring databases [11], $\epsilon$-Spatiotemporal Event Privacy requires the indistinguishability regarding whether the EVENT is true or false given any observations. Another benefit is that, similar to differential privacy whose privacy guarantee is independent of the prior probability of a given databases, the privacy provided by $\epsilon$-Spatiotemporal Event Privacy is independent of the prior probability of the given spatiotemporal even $t^{1}$

Although the privacy goal of spatiotemporal event privacy can be considered as a generalization of location privacy, we note that it may not be true in terms of privacy metrics. We illustrate the indistinguishability-based privacy metrics for the three privacy goals in Fig 3, where the lines connecting two secrets indicate the requirements of indistinguishability between the corresponding two possible values of the secrets.

As shown in Fig 3 (a), indistinguishability-based location privacy metrics (such as geo-indistinguishability [8]) require indistinguishability between each pair of locations. Indistinguishability-based trajectory privacy metrics [9] [12] [13] requires indistinguishability between each pair of possible trajectories as shown in Fig 3 (b). Whereas, $\epsilon$-spatiotemporal event privacy only requires indistinguishability between the defined event and its negation. For example, if the spatiotemporal event is defined as $\operatorname{Pattern}(\mathbf{S}, \mathbf{T})$ where $\mathbf{S}=$ $\left\{\mathbf{s}_{1}, \mathbf{s}_{2}\right\}, \mathbf{s}_{1}=\left\{s_{1}\right\}, \mathbf{s}_{2}=\left\{s_{1}, s_{2}, s_{3}\right\}$ and $\mathbf{T}=\{1,2\}$ (i.e., a trajectory passes through $s_{1}$ and $\left\{s_{1}, s_{2}, s_{3}\right\}$ ) successively, then it does not require the indistinguishability between $\left\{s_{2} \rightarrow\right.$ $\left.s_{3}\right\}$ and $\left\{s_{3} \rightarrow s_{2}\right\}$ as shown in Fig 3 (c). Such privacy requirement makes sense; for example, $s_{1}$ can be a "love

\footnotetext{
${ }^{1}$ In our approach, we first compute the prior probability of the given event with a specific initial distribution $\pi$, and then we make sure that the privacy is guaranteed w.r.t. any $\pi$ in Section IV
}

hotel", $s_{2}$ is "home", and $s_{3}$ is "office". Such spatiotemporal event privacy may not be protected by LPPMs.

While we can define simple events such as a location or trajectory, the corresponding $\epsilon$-spatiotemporal event privacy does not imply the indistinguishability-based location privacy or trajectory privacy. For example, even if a user specifies all possible trajectories as her requirements for $\epsilon$-spatiotemporal event privacy, it only ensures the indistinguishability between "one trajectory" and "not this trajectory", but no guarantee on the indistinguishability between any two trajectories. Hence, the privacy guarantee of $\epsilon$-spatiotemporal event privacy is orthogonal to geo-indistinguishability.

Event-level or user-level differential privacy [14] is different from spatiotemporal event privacy. In such definitions, an event means user's information at a single time point (different to the definition of spatiotemporal event in this paper); hence, eventlevel differential privacy preserves the indistinguishability between any two possible values of a single event. User-level differential privacy preserves the indistinguishability between any two possible sequence of events. These privacy metrics are for time-series data and statistical queries. Despite the difference of the settings, the indistinguishability considered in event-level/user-level differential privacy are similar to the above location privacy and trajectory privacy, which are shown in Fig $3(a)(b)$. Whereas the indistinguishability considered in spatiotemporal event privacy are shown in Fig 3(c).

Location privacy provides general protection against unknown risks when sharing location with the third parties, while spatiotemporal event privacy guarantees flexible and customizable protection which may prevent against profiling attacks such as inferring user's trajectory pattern (location privacy cannot provide such protection). Therefore, it would be preferable that an LPPM achieving location privacy metrics such as geo-indistinguishability also satisfies $\epsilon$-spatiotemporal event privacy. We define $\epsilon$-spatiotemporal event privacy as the indistinguishability between an event and its negation because it provides a clear privacy semantics: the adversaries cannot infer or distinguish whether the event happened or not. Alternatively we can define privacy as indistinguishability between an event and an alternative event. In addition to requiring users to define the alternative events, it presents additional challenges that may involve analyzing the correlations and exclusivity between events and complicated composition property of $\epsilon$-spatiotemporal event privacy. We defer this to future work. 


\section{QUANTIFYING $\epsilon$-SPATIOTEMPORAL EVENT PRIVACY}

\section{A. Overview of our approach}

Given the emission matrix of an LPPM that takes input as user's true location $u^{t}$ and outputs a perturbed location $o^{t}$ at time $t$, quantifying the privacy guarantee under the metric of $\epsilon$-spatiotemporal event privacy is equivalent to calculating the maximum ratio of $\frac{\operatorname{Pr}\left(o_{1}, o_{2}, \cdots, o_{T} \mid \text { EVENT }\right)}{\operatorname{Pr}\left(o_{1}, o_{2}, \cdots, o_{T} \mid \neg \text { EVENT }\right)}$ given any $o_{1}, o_{2}, \cdots, o_{T}$. It is hard to derive this ratio directly from the emission matrix without specifying $o_{1}, o_{2}, \cdots, o_{T}$ and correlations between locations. Firstly, it is because we are not clear about the relationship between the perturbed output $o^{t}$ and the defined EVENT. Secondly, the predicates in the defined EVENT may not be independent. For example, if EVENT is defined as $\left(u^{1}=s_{1}\right) \wedge\left(u^{2}=s_{2}\right)$, the probability of such event, i.e., $\operatorname{Pr}\left(\left(u^{1}=s_{1}\right) \wedge\left(u^{2}=s_{2}\right)\right)$, may not be equal to $\operatorname{Pr}\left(\left(u^{1}=s_{1}\right)\right) * \operatorname{Pr}\left(\left(u^{2}=s_{2}\right)\right)$ because the true locations in user's trajectory can be temporally correlated. For simplicity, we first model the correlation between user's consecutive locations using first-order ${ }^{2}$ time-homogeneous ${ }^{3}$ Markov model, i.e., the transition matrix is identical at each $t$. Then, we quantify this ratio w.r.t. given observations $o_{1}, o_{2}, \cdots, o_{T}$ and a given user's initial probability $\boldsymbol{\pi}$, so that we can directly calculate the $\operatorname{Pr}\left(o_{1}, o_{2}, \cdots, o_{T} \mid\right.$ EVENT $)$. In Section IV we will design a mechanism for spatiotemporal event privacy w.r.t. any observations and arbitrary initial probability. Our goal in this section is to calculate the likelihood of the observations given EvEnT or $\neg$ EvENT , i.e., $\operatorname{Pr}\left(o_{1}, o_{2}, \cdots, o_{T} \mid\right.$ EVENT $)$ or $\operatorname{Pr}\left(o_{1}, o_{2}, \cdots, o_{T} \mid \neg\right.$ EVENT $)$, which can be derived by $\operatorname{Pr}\left(o_{1}, o_{2}, \cdots, o_{T} \mid\right.$ Event $)=\frac{\operatorname{Pr}\left(o_{1}, o_{2}, \cdots, o_{T}, \text { EVENT }\right)}{\operatorname{Pr}(\text { EvenT })}$. We call $\operatorname{Pr}(\mathrm{EVENT})$ as prior probability of the event, and $\operatorname{Pr}\left(o_{1}, o_{2}, \cdots, o_{T}\right.$, EVENT $)$ as joint probability of the event.

One challenge of calculating the prior or joint probabilities of the event is the computational complexity. Given an arbitrary spatiotemporal event, we need to enumerate all possible combination of the Boolean expression for prior and joint probabilities, which can be exponential to the number of predicates in the expression. To address this problem, we propose a two-possible-world method for computing the prior and joint probabilities in Sections III-B and III-C, respectively.

For ease of exposition, we define notations frequently used in the following sections. $\mathbf{M} \in \mathbb{R}^{m \times m}$ denotes a transition matrix that describes temporal correlations in user's location. At timestamp 1, an initial probability is denoted by $\pi \in[0,1]^{1 \times m}$. During timestamp $\{1,2, \cdots, T\}$, the probability of the true location $\operatorname{Pr}\left(u^{t}\right)$ is denoted by a row vector $\mathbf{p}_{t} \in[0,1]^{1 \times m}$ where the $i$ th element denotes $\operatorname{Pr}\left(u^{t}=s_{i}\right)$. A Markov model follows the transition property of $\mathbf{p}_{t+1}=\mathbf{p}_{t} \mathbf{M}$, e.g., after a Markov transition, $\mathbf{p}_{2}=\boldsymbol{\pi} \mathbf{M}$ at timestamp 2 given $\mathbf{p}_{1}=\boldsymbol{\pi}$.

The notations below for matrix computation are also used in the rest of this paper. Let $\mathbf{0}$ and $\mathbf{1}$ be row vectors with $m$ elements being 0 and 1 respectively. $[\mathbf{0}, \mathbf{1}]$ is a row vector in

\footnotetext{
${ }^{2}$ If the Markov model is high-ordered, i.e., the transition matrix has a larger state domain, our approach still works by applying the new matrix.

${ }^{3}$ If the Markov model is time-varying, i.e., transition matrices at different $t$ are not identical, our approach still works. We explain this in the next section.
}

$\mathbb{R}^{1 \times 2 m} \cdot \mathbf{a} \circ \mathbf{b}$ denotes the Hadamard product of $\mathbf{a}$ and $\mathbf{b} . \mathbf{a}^{\mathbf{D}}$ is a diagonal matrix with the elements of vector $\mathbf{a}$ on the diagonal.

\section{B. Computing Prior Probability of an Event}

To avoid the exponential complexity, we propose an efficient algorithm with two possible worlds. The idea is to elaborate a "new" transition matrix $\mathbf{M}_{t} \in \mathbb{R}^{2 m \times 2 m}$ at each time $t$ which encodes the complex spatiotemporal event inside, so that the calculation of the prior or joint probability for a complicated event is the same as one simple predicate.

Intuition. The main idea of our method is to use two virtual worlds denoting whether the EVENT is true or false. The states in the two worlds denote the joint probabilities $\operatorname{Pr}\left(u^{t}=\right.$ $s_{i}$, EvEnt $)$ and $\operatorname{Pr}\left(u^{t}=s_{i}, \neg\right.$ EVEnt $)$. For PrESEnCE, once a trajectory enters into the region of the PRESENCE, its probability will be kept in the world of true EVENT forever. For PATTERN, the probability distribution among the two worlds are derived at the beginning timestamp of the EVENT, and only the trajectories satisfying the PATTERN will be kept in the world of true EVENT. At last, the sum of probabilities in the world of true EVENT will be $\operatorname{Pr}($ EVENT is true).

Presence. We first study the Presence event. Let us consider the following example.

Example III.1. Let $\mathbb{S}=\left\{s_{1}, s_{2}, s_{3}\right\}$. A PRESENCE event is defined in $s_{1}$ and $s_{2}$ during $t=3$ and $t=4$, i.e., $\boldsymbol{s}=[1,1,0]^{\top}$, start $=3$, end $=4$. The transition matrix $\boldsymbol{M}$ is given below.

$$
\boldsymbol{M}=\left[\begin{array}{ccc}
0.1 & 0.2 & 0.7 \\
0.4 & 0.1 & 0.5 \\
0 & 0.1 & 0.9
\end{array}\right]
$$

Then Fig. 4 shows the new transitions in the two worlds, the top world and the bottom world separated by the dashed line in Fig.4. From time 1 to 2, a normal transition can be made. At timestamp 2, all the transitions going to the states $s_{1}$ and $s_{2}$ will be re-directed to the new states $s_{1}^{\prime}$ and $s_{2}^{\prime}$, denoting the states when the PRESENCE happens. Other transitions that do not go the the area will perform normally. Similarly at time 3 , the transition from $s_{3}$ to $s_{2}$ will also go to the state $s_{2}^{\prime}$ because the event is also true in this case. After time 4, the original Markov transitions come back to work again.

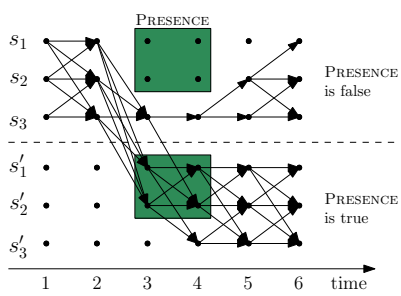

Fig. 4: New Markov transitions: all transitions going to the PRESENCE area will be re-directed to the virtual worlds.

The intuition can be formalized as follows. First, the original probabilities in $\mathbb{R}^{1 \times m}$ is extended to $\mathbb{R}^{1 \times 2 m}$. Thus the initial probability $\boldsymbol{\pi}$ becomes $[\boldsymbol{\pi}, \mathbf{0}]$. Second, the transition matrix $\mathbf{M}_{t}$ becomes the form of four transition matrices between the two virtual worlds, i.e. the EVENT is true or false, in Equation (3). Then the new transition matrices can be derived in Equations (4) and (5) where $\mathbf{M}$ is the original transition matrix and $\mathbf{S}$ is the region of PRESENCE defined in Definition II.2 


$$
\begin{gathered}
\mathbf{M}_{t}=\left[\begin{array}{cc}
\text { false } \rightarrow \text { false } & \text { false } \rightarrow \text { true } \\
\text { true } \rightarrow \text { false } & \text { true } \rightarrow \text { true }
\end{array}\right] \text { on the event. } \\
\mathbf{M}_{t}=\left[\begin{array}{cc}
\mathbf{M}-\mathbf{M s}^{\mathbf{D}} & \mathbf{M s}^{\mathbf{D}} \\
\mathbf{0}^{\mathbf{D}} & \mathbf{M}
\end{array}\right], \text { start }-1 \leq t \leq \text { end }-1 . \\
\mathbf{M}_{t}=\left[\begin{array}{cc}
\mathbf{M} & \mathbf{0}^{\mathbf{D}} \\
\mathbf{0}^{\mathbf{D}} & \mathbf{M}
\end{array}\right], t<\text { start }-1 \text { or } t \geq \text { end } .
\end{gathered}
$$

Equation (4), designed to capture and maintain all the transitions going to the region of the PRESENCE, is the new transition matrix when entering (and inside) the event time. Equation (5), designed to keep the original transitions in the two virtual worlds, is the new transition matrix when leaving (and before) the event time. Third, at the last time $T$, the probability of the PRESENCE will be the sum of all probabilities in the bottom world (where PRESENCE is true).

Pattern. For PATtern events, the bottom world denoting the event is true only needs to preserve the transitions going to the defined areas of the PATTERn event. The following example shows the mechanism.

Example III.2. We study the PATTERN event in Fig.5. At time 1 , the transitions entering $s_{1}$ and $s_{2}$ go to $s_{1}^{\prime}$ and $s_{2}^{\prime}$. From time 2 to 4 , the transitions in the above world perform normally. But the transitions from the bottom world go back to the top world if the destinations are not in the defined regions. At time 5 , the original Markov transitions come back to world again.

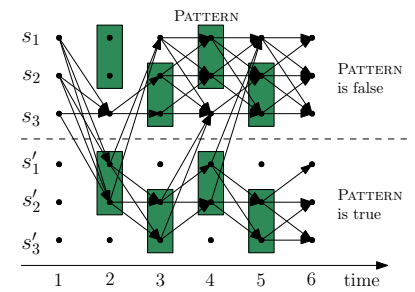

Fig. 5: New Markov transitions: at timestamp 1, all transitions going to the defined area will be re-directed to the bottom world; at timestamp $2 \sim 4$, only the transitions from the bottom world to the defined areas remain below.

From above example, the transition matrix for PATTERN only differs from that for PRESENCE during the event time, shown in Equation (7) where $\mathbf{s}_{t}$ is the regions defined in Definition III.3. The transitions outside the event time, i.e. $t<$ start or $t \geq e n d$, will be the same as Equations (4) and (5).

$$
\begin{aligned}
& \mathbf{M}_{t}=\left[\begin{array}{cc}
\mathbf{M}-\mathbf{M s}^{\mathbf{D}} & \mathbf{M} \mathbf{s}^{\mathbf{D}} \\
\mathbf{0}^{\mathbf{D}} & \mathbf{M}
\end{array}\right], t=\text { start }-1 . \\
& \mathbf{M}_{t}=\left[\begin{array}{cc}
\mathbf{M} & \mathbf{0}^{\mathbf{D}} \\
\mathbf{M}-\mathbf{M} \mathbf{s}_{t}^{\mathbf{D}} & \mathbf{M s}_{t}^{\mathbf{D}}
\end{array}\right], \text { start } \leq t \leq \text { end }-1 . \\
& \mathbf{M}_{t}=\left[\begin{array}{ll}
\mathbf{M} & \mathbf{0}^{\mathbf{D}} \\
\mathbf{0}^{\mathbf{D}} & \mathbf{M}
\end{array}\right], t<\text { start }-1 \text { or } t \geq \text { end } .
\end{aligned}
$$

In summary, the prior probability of any EVENT can be derived as the sum of probabilities in the world where the EVENT is true. Lemma III.1 shows the formal computation.

Lemma III.1. For initial probability $\boldsymbol{\pi} \in \mathbb{R}^{1 \times m}$, the prior probability of an EVENT of PRESENCE and PATTERN is

$$
\operatorname{Pr}(\text { Event })=[\boldsymbol{\pi}, \boldsymbol{0}] \prod_{i=1}^{e n d-1} \boldsymbol{M}_{i}[\boldsymbol{0}, \boldsymbol{1}]^{\top}
$$

where $\boldsymbol{M}_{i}$ is computed by Equations (4), (5), (6), (7), (8).

When the transition matrices $\mathbf{M}$ at different $t$ are not identical, the only extra effort is to re-compute Equations (4) (8) using the corresponding transition matrix $\mathbf{M}$ at $t$.

\section{Computing Joint Probability of an Event}

The calculation of a spatiotemporal event and a set of observed locations, i.e, $\operatorname{Pr}\left(o_{1}, o_{2}, \cdots, o_{T}\right.$, EVENT $)$ is a little more complicated than previous sections since it dependents on not only the initial probabilities but also the emission matrix of the LPPM. Similarly, we use two-possible-world method to avoid enumerating all possible cases of an event. We utilize forward-backward algorithm [15] to estimate the probability of the true state (true location) at timestamp $t$ given all observations $\operatorname{Pr}\left(u^{t} \mid o_{1}, o_{2}, \cdots, o_{T}\right)$. It first calculates a forward probability $\alpha_{t}^{k}=\operatorname{Pr}\left(u^{t}=s_{k}, o_{1}, o_{2}, \cdots, o_{t}\right)$ iteratively, i.e.,

$$
\alpha_{t}^{k}=\operatorname{Pr}\left(o_{t} \mid u^{t}=s_{k}\right) \sum_{i} \alpha_{t-1}^{i} \operatorname{Pr}\left(u^{t}=s_{k} \mid u_{t-1}=s_{i}\right) .
$$

Then, a backward probability $\beta_{t}^{k}=\operatorname{Pr}\left(o_{t+1}, o_{t+2}, \cdots, o_{T} \mid u^{t}=\right.$ $s_{k}$ ) can also be derived by

$$
\beta_{t}^{k}=\sum_{i} \operatorname{Pr}\left(u_{t+1}=s_{i} \mid u^{t}=s_{k}\right) \operatorname{Pr}\left(o_{t+1} \mid u_{t+1}=s_{i}\right) \beta_{t+1}^{i} .
$$

By initializing $\beta_{T}^{k}=1$ for all $k$, we can obtain the estimation of $u^{t}$ as follows.

$$
\operatorname{Pr}\left(u^{t}=s_{k} \mid o_{1}, o_{2}, \cdots, o_{T}\right)=\frac{\alpha_{t}^{k} \beta_{t}^{k}}{\sum_{i} \alpha_{t}^{i} \beta_{t}^{i}}
$$

Intuition. The intuition of our solution is to use the forwardbackward algorithm in the two virtual worlds where the Event is true and false. This is feasible because the emission probability, which determines the probabilities of the observations, is independent from any Events. Hence in our computation the forward probability and backward probability are $\operatorname{Pr}\left(\right.$ EVENT $\left., o_{1}, o_{2}, \cdots, o_{t}\right)$ for $t \leq$ end and $\operatorname{Pr}\left(o_{e n d+1}, o_{e n d+2}, \cdots, o_{t} \mid\right.$ EVENT $)$ for $t>$ end respectively. By combining them together, we can obtain the posterior probability of the EVENT. Note that at any timestamp $t \leq e n d$, we do not see the future $(t>e n d)$ observations. Thus the posterior probability only counts to the current timestamp $t$.

Before and During the Event. In the forward algorithm, the probability $\alpha_{t}^{k}=\operatorname{Pr}\left(u^{t}=s_{k}, o_{1}, o_{2}, \cdots, o_{t}\right)$ is derived at timestamp $t$. We represent $\alpha_{t}^{k}$ in the vector form $\boldsymbol{\alpha}_{t}=\left[\alpha_{1}^{1}, \alpha_{t}^{2}, \cdots, \alpha_{t}^{m}\right]$. Then it can be derived as $\boldsymbol{\alpha}_{t}=$ $\left(\boldsymbol{\alpha}_{t-1} \mathbf{M}_{t-1}\right) \circ \tilde{\mathbf{p}}_{o_{t}}=\boldsymbol{\alpha}_{t-1} \mathbf{M}_{t-1} \tilde{\mathbf{p}}_{o_{t}}^{\mathbf{D}}$. Without any further observations, the joint probability can be derived from Lemma III.1. The result is shown in Lemma III.2

Lemma III.2. Given initial probability $\boldsymbol{\pi} \in \mathbb{R}^{1 \times m}$, the joint probability of an EVENT of PRESENCE or PATTERN and observations $o_{1}, o_{2}, \cdots, o_{t}$ at any timestamp $t \leq$ end is 
$\operatorname{Pr}\left(\right.$ Event $\left., o_{1}, o_{2}, \cdots, o_{t}\right)=[\boldsymbol{\pi}, \boldsymbol{0}]\left(\tilde{\boldsymbol{p}}_{o_{1}}^{\boldsymbol{D}} \prod_{i=2}^{t}\left(\boldsymbol{M}_{i-1} \tilde{\boldsymbol{p}}_{o_{i}}^{\boldsymbol{D}}\right) \prod_{i=t}^{e n d-1} \boldsymbol{M}_{i}[\boldsymbol{0}, \boldsymbol{I}]^{\boldsymbol{\top}}\right)$

After the Event. In the backward algorithm, $\beta_{t}^{k}=$ $\operatorname{Pr}\left(o_{t+1}, o_{t+2}, \cdots, o_{T} \mid u^{t}=s_{k}\right)$. We represent it in the vector form $\boldsymbol{\beta}_{t}=\left[\beta_{t}^{1}, \beta_{t}^{2}, \cdots, \beta_{t}^{m}\right]$. Then it can be derived as $\boldsymbol{\beta}_{t}=$ $\left(\boldsymbol{\beta}_{t+1} \circ \tilde{\mathbf{p}}_{o_{t+1}}\right) \mathbf{M}_{t}^{\boldsymbol{\top}}=\boldsymbol{\beta}_{t+1} \tilde{\mathbf{p}}_{o_{t+1}}^{\mathbf{D}} \mathbf{M}_{t}^{\boldsymbol{\top}}$ for any $t>$ end. Similarly, the joint probability can be obtained by Lemma III.3

Lemma III.3. Given initial probability $\pi \in \mathbb{R}^{1 \times m}$, the joint probability of an EVENT of PRESENCE or PATTERN and observations $o_{1}, o_{2}, \cdots, o_{t}$ at any timestamp $t>$ end is

$$
\begin{aligned}
& \operatorname{Pr}\left(\text { Event, } o_{1}, o_{2}, \cdots, o_{t}\right)=[\boldsymbol{\pi}, \boldsymbol{0}] \\
& \left(\tilde{\boldsymbol{p}}_{o_{1}}^{D} \prod_{i=2}^{\text {end }}\left(\boldsymbol{M}_{i-1} \tilde{\boldsymbol{p}}_{o_{i}}^{\boldsymbol{D}}\right)\right)\left([\boldsymbol{I}, \boldsymbol{I}] \prod_{i=t-1}^{\text {end }}\left(\tilde{\boldsymbol{p}}_{o_{i+1}}^{\boldsymbol{D}} \boldsymbol{M}_{i}^{\boldsymbol{\top}}\right) \circ[\boldsymbol{0}, \boldsymbol{I}]\right)^{\top}
\end{aligned}
$$

To summarize, now we can quantify the ratio $\operatorname{Pr}\left(o_{1}, o_{2}, \cdots, o_{T} \mid\right.$ EVENT $)=\frac{\operatorname{Pr}\left(o_{1}, o_{2}, \cdots, o_{T}, \text { EVENT }\right)}{\operatorname{Pr}(\text { EVENT })}$ for spatiotemporal event privacy using Lemma III.1 to compute $\operatorname{Pr}($ EVENT) and Lemmas III.2, III.3 to compute $\operatorname{Pr}\left(o_{1}, o_{2}, \cdots, o_{T}\right.$, EVENT $)$. We note that our approach of computing the joint probability of an event is able to deal with different emission matrices at each $t$. Since $\tilde{\mathbf{p}}_{o_{t}}$ is a vector of emission probabilities given the observation $o_{t}$, i.e, a column in the emission matrix, and $\tilde{\mathbf{p}}_{o_{t}}^{\mathbf{D}}$ is a diagonal matrix whose diagonal elements are $\tilde{\mathbf{p}}_{o t}$, we only need to obtain $\tilde{\mathbf{p}}_{o t}$ and $\tilde{\mathbf{p}}_{o_{t}}^{\mathbf{D}}$ from the corresponding emission matrix at $t$, and then use such $\tilde{\mathbf{p}}_{o_{t}}^{\mathbf{D}}$ in Equations (13) and (14).

\section{PRISTE FRAMEWORK}

In previous section, we designed methods for quantifying $\epsilon$-spatiotemporal event privacy w.r.t. a specified initial probability, which means that the quantification may not be true if attacker has a different initial probability since different initial probabilities may result in very different prior and joint probabilities. In this section, we first solve this problem to ensure $\epsilon$ spatiotemporal event privacy for any initial probabilities, then design a framework that converts planar Laplace mechanism into one protecting spatiotemporal event privacy.

\section{A. Tackling Arbitrary Initial Probability}

According to the quantification in Section III we can calculate $\frac{\operatorname{Pr}\left(o_{1}, o_{2}, \cdots, o_{T} \mid \text { EVENT }\right)}{\operatorname{Pr}\left(o_{1}, o_{2}, \cdots, o_{T} \mid \neg \text { EVENT }\right)}$ given $o_{1}, o_{2}, \cdots, o_{T}$ and a given initial probability $\boldsymbol{\pi}$. In the next section, we will propose a framework that ensures any output from the framework satisfies $\epsilon$-spatiotemporal event privacy. In this section, we show how to make sure the ratio is bounded given arbitrary initial probability. Our idea to is taking $\boldsymbol{\pi}$ as a variable and solving the maximization problem of $\frac{\operatorname{Pr}\left(o_{1}, o_{2}, \cdots, o_{T} \mid \text { EVENT }\right)}{\operatorname{Pr}\left(o_{1}, o_{2}, \cdots, o_{T} \mid \neg \text { EVENT }\right)}-e^{\epsilon}$. We want to make sure the maximum value is always less than or equal to 0 , i.e., the user enjoys plausible deniability for her specified spatiotemporal event.

The following theorem shows the conditions related to $\pi$ that satisfies $\epsilon$-spatiotemporal event privacy. We will formulate it as an optimization problem.

Theorem IV.1. For an EVENT of PRESENCE or PATTERN and an arbitrary initial probability $\boldsymbol{\pi} \in \mathbb{R}^{1 \times m}$, $\epsilon$ spatiotemporal event privacy is satisfied at any timestamp $t$, i.e., $\frac{\operatorname{Pr}\left(o_{1}, o_{2}, \cdots, o_{T} \mid \mathrm{EVENT}\right)}{\operatorname{Pr}\left(o_{1}, o_{2}, \cdots, o_{T} \mid \neg \mathrm{EVENT}\right)} \leq e^{\epsilon}$, if the observation $o_{t}$ is released based on the following two conditions

$$
\begin{aligned}
& \boldsymbol{\pi}\left(\left[\boldsymbol{I}^{\boldsymbol{D}}, \boldsymbol{0}^{\boldsymbol{D}}\right]\left(\left(e^{\epsilon}-1\right) \boldsymbol{a}^{\top} \boldsymbol{b}-e^{\epsilon} \boldsymbol{a}^{\boldsymbol{\top}} \boldsymbol{c}\right)\left[\boldsymbol{I}^{\boldsymbol{D}}, \boldsymbol{0}^{\boldsymbol{D}}\right]^{\boldsymbol{\top}}\right) \boldsymbol{\pi}^{\boldsymbol{\top}}+\boldsymbol{\pi}\left[\boldsymbol{I}^{\boldsymbol{D}}, \boldsymbol{0}^{\boldsymbol{D}}\right]\left(\boldsymbol{b}^{\boldsymbol{\top}}\right) \leq 0 \\
& \boldsymbol{\pi}\left(\left[\boldsymbol{I}^{\boldsymbol{D}}, \boldsymbol{0}^{\boldsymbol{D}}\right]\left(\left(e^{\epsilon}-1\right) \boldsymbol{a}^{\top} \boldsymbol{b}+\boldsymbol{a}^{\top} \boldsymbol{c}\right)\left[\boldsymbol{I}^{\boldsymbol{D}}, \boldsymbol{0}^{\boldsymbol{D}}\right]^{\top}\right) \boldsymbol{\pi}^{\boldsymbol{\top}}-\boldsymbol{\pi}\left[\boldsymbol{I}^{\boldsymbol{D}}, \boldsymbol{0}^{\boldsymbol{D}}\right]\left(e^{\epsilon} \boldsymbol{b}^{\top}\right) \leq 0
\end{aligned}
$$

where

$$
\boldsymbol{a}^{\boldsymbol{\top}}=\prod_{i=1}^{e n d-1} \boldsymbol{M}_{i}[\boldsymbol{0}, \boldsymbol{I}]^{\top}
$$

For $t \leq e n d$,

$$
\boldsymbol{b}^{\boldsymbol{\top}}=\tilde{\boldsymbol{p}}_{o_{1}}^{\boldsymbol{D}} \prod_{i=2}^{t}\left(\boldsymbol{M}_{i-1} \tilde{\boldsymbol{p}}_{o_{i}}^{\boldsymbol{D}}\right) \prod_{i=t}^{e n d-1} \boldsymbol{M}_{i}[\boldsymbol{0}, \boldsymbol{I}]^{\top} \boldsymbol{c}^{\boldsymbol{\top}}=\tilde{\boldsymbol{p}}_{o_{1}}^{\boldsymbol{D}} \prod_{i=2}^{t}\left(\boldsymbol{M}_{i-1} \tilde{\boldsymbol{p}}_{o_{i}}^{\boldsymbol{D}}\right)[\boldsymbol{I}, \boldsymbol{I}]^{\top}
$$

For $t>$ end,

$$
\begin{aligned}
& \boldsymbol{b}^{\boldsymbol{\top}}=\tilde{\boldsymbol{p}}_{o_{1}}^{\boldsymbol{D}} \prod_{i=2}^{e n d}\left(\boldsymbol{M}_{i-1} \tilde{\boldsymbol{p}}_{o_{i}}^{\boldsymbol{D}}\right)\left([\boldsymbol{l}, \boldsymbol{1}] \prod_{i=t-1}^{e n d}\left(\tilde{\boldsymbol{p}}_{o_{i+1}}^{\boldsymbol{D}} \boldsymbol{M}_{i}^{\boldsymbol{\top}}\right) \circ[\boldsymbol{0}, \boldsymbol{1}]\right)^{\top} \\
& \boldsymbol{c}^{\boldsymbol{\top}}=\tilde{\boldsymbol{p}}_{o_{1}}^{\boldsymbol{D}} \prod_{i=2}^{e n d}\left(\boldsymbol{M}_{i-1} \tilde{\boldsymbol{p}}_{o_{i}}^{\boldsymbol{D}}\right)\left([\boldsymbol{1}, \boldsymbol{1}] \prod_{i=t-1}^{e n d}\left(\tilde{\boldsymbol{p}}_{o_{i+1}}^{\boldsymbol{D}} \boldsymbol{M}_{i}^{\boldsymbol{\top}}\right) \circ[\boldsymbol{1}, \boldsymbol{l}]\right)^{\top}
\end{aligned}
$$

Quadratic Programming. To determine whether Equations (15) and (16) are true or not for arbitrary $\pi$, we transform them to maximization problems: finding the maximum values of the left parts of Equations (15) and (16) under the constraints of $0 \leq p_{i} \leq 1$ where $p_{i} \in \boldsymbol{\pi}$. As long as one of maximum values is larger than 0 , then we know that the LPPM (emission matrix) may not satisfy $\epsilon$-spatiotemporal event privacy. The maximization are equivalent to quadratic programing problem since they can be rewritten in a form of $\boldsymbol{\pi} \mathbf{A} \boldsymbol{\pi}^{\top}=\frac{1}{2} \boldsymbol{\pi}\left(\mathbf{A}+\mathbf{A}^{\top}\right) \boldsymbol{\pi}^{\top}$ where $\mathbf{A}$ is a matrix. We skip the computation details about solving such quadratic programing problem since many methods and tools have been proposed in literature. In experiments, we use IBM CPLEX optimizer as our computation engine.

\section{B. PriSTE}

Based on the quantification techniques that we developed in previous sections, we propose a framework that converts a location privacy protection mechanism into one protecting spatiotemporal event privacy. The PriSTE framework is illustrated in Fig 6 and described in Algorithm 1 .

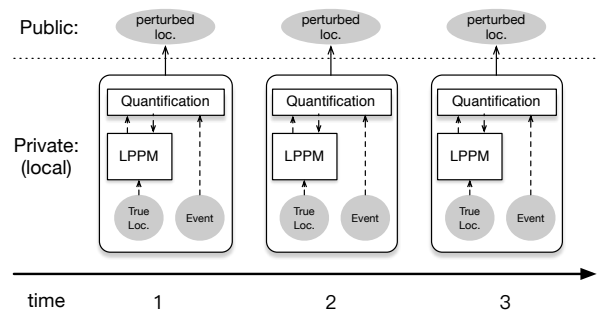

Fig. 6: PriSTE framework

The major components are Quantification and a given LPPM. Their interactions are described as follows. First, the LPPM generates a perturbed location from the true location and pass it to the Quantification component. By Theorem IV.1 the Quantification component check whether this perturbed location satisfies the ratio in Equation (1) ( $\epsilon$-spatiotemporal 
event privacy) or not, under a sequence of previous observations and user-specified spatiotemporal events. If not, we need to calibrate the emission matrix to ensure that it satisfies $\epsilon$-spatiotemporal event privacy. The strategy of emission matrix calibration is LPPM-dependent. In the next section, we demonstrate a case study for Planar Laplace Mechanism [8], which is the state-of-the-art mechanism for location privacy.

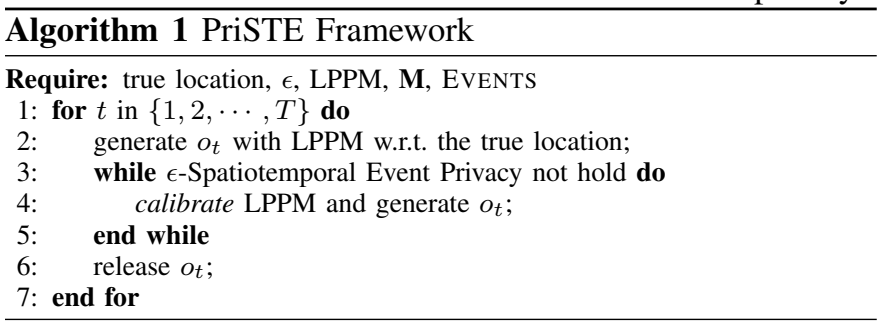

\section{Case Study 1: PriSTE with Geo-indistinguishability.}

In this section, we instantiate PriSTE framework using $\alpha$ Planar Laplace mechanism ( $\alpha$-PLM) which is designed for Geo-indistinguishability [8]. We first show the computation details for quantifying $\epsilon$-spatiotemporal event privacy by Theorem IV.1 and then design a greedy strategy for approximately achieving $\epsilon$-spatiotemporal event privacy.

Algorithm Design. To implement the quantification component, we need to (1) compute the internal parameters $\mathbf{a}, \mathbf{b}$ and c shown in Theorem IV.1 and (2) design a strategy to calibrate the emission matrix.

As for the calibration strategy for Planar Laplace Mechanism (PLM) with a specified privacy budget $\alpha$ (which solely determine the shape of the output distribution), we exponentially decay its privacy budget because the smaller privacy budget implies stronger protection for location privacy and less information disclosure. In our algorithm, decay rate $\frac{1}{2}$ for the privacy budget in line 10 of Algorithm 2 is a tunable parameter that provides a trade-off between efficiency and utility of the released locations. Setting a small value allows the algorithm converge faster, but at the cost of over-perturbing the location at each timestamp. In contrast, using a large value is less efficient but allows better utility to be achieved.

A natural question is that can we always find an $\alpha$ to release a perturbed location that satisfies Equation (1). The answer is yes because $\alpha$ converges exponentially to 0 . When $\alpha=$ 0 , it releases no useful information about the true location, i.e., uniformly returning a location without using user's true position. It is easy to verify that the Equations (15) and (16) are always true in this situation.

Algorithm 2 shows the computation process. To boost the efficiency of our algorithm, we use intermediate matrices A and $\mathbf{B}$ to facilitate the computation of $\mathbf{b}$ and $\mathbf{c}$. At time 1 , we initialize the variables as line $4 \sim 8$. At any time before and inside the EVENT, we compute the variables as line $10 \sim 11$. At any timestamps after the EVENT, the variables are derived as line $13 \sim 14$. Then we use quadratic programming methods to check Eq. (15) and (16) to decide whether to release the $o_{t}$ or not. If not, we generate a new $o_{t}$ with only half $\alpha$, and repeat the above process again. Finally, we update the matrices $\mathbf{A}$ and
$\mathbf{B}$ as line 21 25. If $t=e n d$, in line 10 the product $\prod_{i=t}^{e n d-1} \mathbf{M}_{i}$ will be the identity matrix. In line $22, \mathbf{M}_{0}$ is the identity matrix when $t=1$. We note that for multiple EvENTs, Algorithm 2 can be executed multiple times for each EVENT.

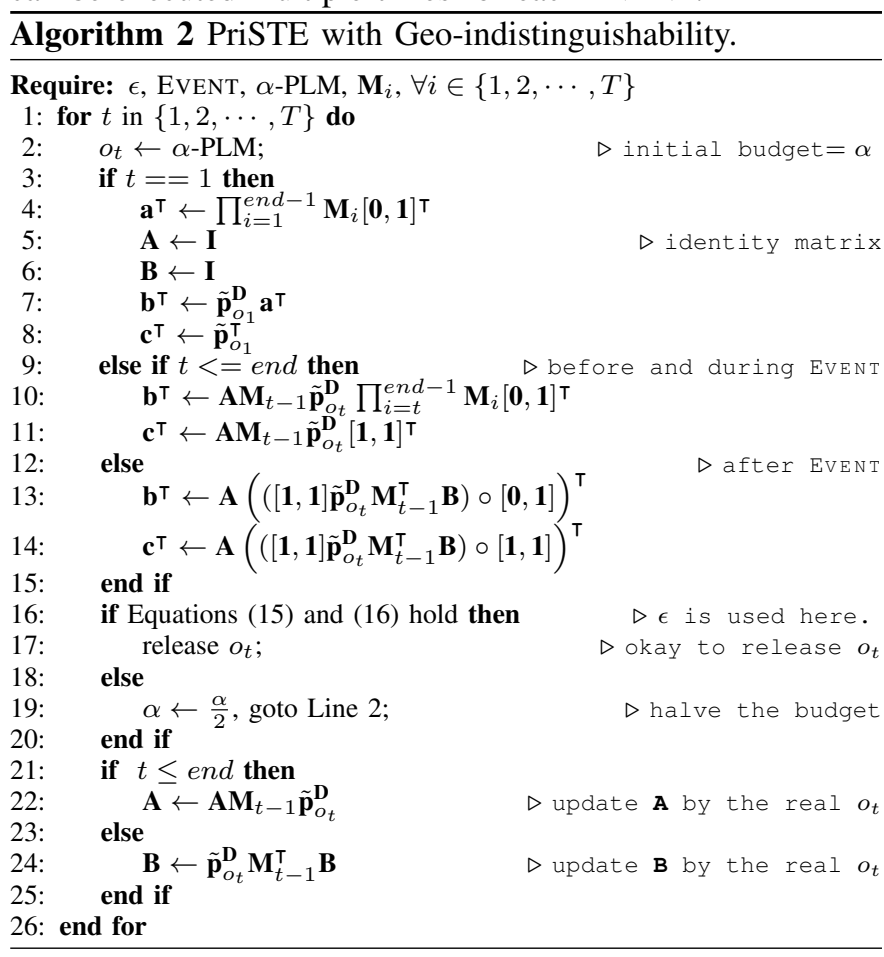

Complexity. The internal parameters $\mathbf{a}, \mathbf{b}$ and $\mathbf{c}$ in Algorithm 2 need $O(m T)$ time to be evaluated. The major computational cost lies in the quadratic program for checking Equations (15) and (16). The complexity will be determined by the quadratic matrix $\left[\mathbf{1}^{\mathbf{D}}, \mathbf{0}^{\mathbf{D}}\right] \mathbf{a}^{\top} \mathbf{c}\left[\mathbf{1}^{\mathbf{D}}, \mathbf{0}^{\mathbf{D}}\right]^{\boldsymbol{T}}$. If it is positive definite, then the complexity is $O\left(\mathrm{~m}^{3}\right)$. Otherwise, with any negative eigenvalues, it will be NP-hard [16]. In our experiments, we use IBM CPLEX which can provide globally optimal results for quadratic program but may need a long computation time. We use a conservative release strategy to remedy this: we use a threshold to limit the computation time of quadratic program for checking Eq. (15) and Eq.(16). It will not release a perturbed location unless we are sure that the equations are true. Although it may lead to suboptimal solution in budget calibration, it always guarantees $\epsilon$-spatiotemporal event privacy since every released locations satisfy Eq. [15) and (16). Privacy Analysis. PriSTE framework relies on a local model, i.e., the assumption that adversaries cannot obtain user's locally stored information as shown in Fig 6 . Although line 2 may be executed more than once at a time point $t$, Algorithm 2 still satisfies $\alpha^{\prime}$-geo-indistinguishability where $\alpha^{\prime}$ is the final privacy budget used for releasing $o_{t}$ because that is the only observation of attacker at time $t$. If we remove the assumption of local model, the above statements may not be true since attacker may observe the internal states of the algorithm (which is the privacy goal of pan-privacy [17]). Examples of internal states includes multiple $o_{t}$ tested at $t$ or the final $\alpha^{\prime}$ used in the algorithm. Anothe assumption that may affect the privacy guarantee is the transition matrix $\mathbf{M}$, which we use it 
to model the correlations between locations and assume that it is given. It is an interesting future work to quantify the change of privacy loss in terms of $\epsilon$-spatiotemporal event privacy if the ground truth of correlation is not the modeled one. We defer this study to future work.

\section{Case Study 2: PriSTE with $\delta$-Location Set Privacy.}

To demonstrate the effectiveness of PriSTE framework, we also instantiate it using another privacy metric $\delta$-location set privacy [9] [18], which is proposed for obtaining better utility by taking advantage of temporal correlation between consecutive locations in user's trajectory. The key idea is that hiding the true location in any impossible locations (e.g., whose probabilities are close to 0 ) is a lost cause because the adversary already knows the user cannot be there. In other words, it restricts the output domain of the emission matrix to $\delta$-location set, which is a set containing minimum number of locations that have prior probability sum no less than $1-\delta$. A larger $\delta$ indicates weaker privacy guarantee.

The privacy metrics of $\alpha$-geo-indistinguishability and $\delta$ location set privacy are orthogonal because the former requires a specific "shape" of emission distribution and the latter restricts output domain of the emission distribution. In [9], Xiao and Xiong proposed a framework to achieve $\delta$-location set privacy using a given LPPM. For ease of comparison, we use $\alpha$-LPM as the underlying LPPM for $\delta$-location set privacy.

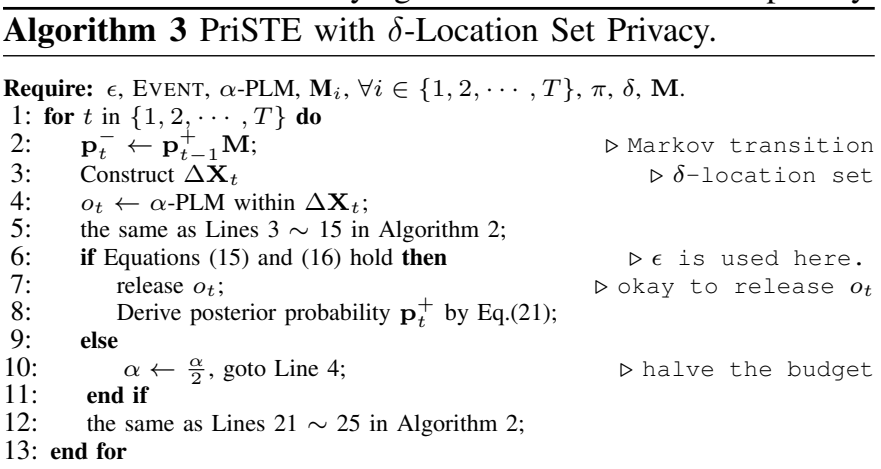

In Line 2, when $t=1$, we have $\mathbf{p}_{0}^{+}=\pi$. In Line 8 , according to [9], the posterior probability can be calculated by Equation (21) where $\mathbf{p}_{t}^{+}[j]$ and $\mathbf{p}_{t}^{-}[i]$ are the $i$ th elements in the corresponding probability vectors.

$$
\mathbf{p}_{t}^{+}[i]=\operatorname{Pr}\left(u^{t}=s_{i} \mid o_{t}\right)=\frac{\operatorname{Pr}\left(o_{t} \mid u^{t}=s_{i}\right) * \mathbf{p}_{t}^{-}[i]}{\sum_{j} \operatorname{Pr}\left(o_{t} \mid u^{t}=s_{j}\right) * \mathbf{p}_{t}^{-}[j]}
$$

The calculation of $\delta$-location set requires the initial probability $\pi$. In experiments, we set $\pi$ to a uniform distribution.

\section{EXPERIMENTAL EVALUATION}

In experiments, we verified that Algorithms 2 and 3 can adaptively calibrate the privacy budget of Planar Laplace Mechanism (PLM) at each timestamp for $\epsilon$-spatiotemporal event privacy. We highlight the following empirical findings.

- A stricter LPPM can satisfy a certain level of spatiotemporal event privacy without any change, whereas a more loose LPPM may need to reduce its privacy budget significantly for protecting the same event.
- For achieving a specific $\epsilon$-spatiotemporal event privacy, a stricter LPPM is not always better in terms of data utility.

- If the user's transition matrix has a significant pattern, an LPPM may need a small privacy budge to achieve $\epsilon$-spatiotemporal event privacy.

\section{A. Experiment Settings and Metrics}

Dataset. We used real-life and synthetic datasets in experiments. Geolife data [19] was collected from 182 users in a period of over three years. It recorded a wide range of users' outdoor movements, represented by a series of tuples containing latitude, longitude and timestamp. The user's entire trajectory is used to train the transition matrix $\mathbf{M}$, e.g. with $\mathrm{R}$ package "markovchain".

We generated a synthetic trajectory and its transition probability matrix as follows. First, a map with $20 * 20$ cells is generated. Then, the transition probability from one cell to another is proportional to the two-dimensional Gaussian distribution with scale parameter $\sigma$. Here, a smaller $\sigma$ indicates that the user moves to the adjacent cells in a higher probability, i.e., the transition matrix has a more significant pattern. Finally, we produced trajectories with 50 timestamps using such transition matrix to simulate movement of a user.

Quadratic Programming. We use the IBM CPLEX optimizer $^{4}$ to find the globally optimal solution for the quadratic programming in Algorithm 2. We adopt a strategy of conservative release as mentioned previously and limit the computation time for each optimization to 1 seconds.

Events. We investigate Presence and PATtern events, which are represented by two parameters $\mathbf{S}$ and $\mathbf{T}$. For example, $\operatorname{Presence}(\mathbf{S}=\{1: 10\}, \mathbf{T}=\{4: 8\})$ is Presence event denoting the user appears in the region of $\left\{s_{1}, s_{2}, \cdots, s_{10}\right\}$ during timestamps $\{4,5,6,7,8\}$.

Utility Metrics. We use two metrics to evaluate data utility.

- Privacy budget $\alpha$ used in PLM, including $\alpha$ at each timestamp (see Section $\mathrm{V}$-B and the average $\alpha$ during the whole time period (see Section $\mathrm{V}-\mathrm{C}$ ). The higher privacy budget indicates the higher utility.

- The Euclidean distance between the perturbed locations and the true locations. The smaller Euclidean distance indicates the higher utility.

We run our algorithm 100 times and aggregate the results to calculate average privacy budget and Euclidean distance.

\section{B. Utility at Each Timestamp}

In this section, we show the utility (average privacy budget over 100 runs) at each timestamp for protecting $\operatorname{PrESENCE}(\mathbf{S}=$ $\{1: 10\}, \mathbf{T}=\{4: 8\})$ and $\operatorname{Presence}(\mathbf{S}=\{1: 10\}, \mathbf{T}=\{16: 20\})$.

PriSTE with Geo-indistinguishability. In Fig 7(a), it turns out that, 0.2-PLM satisfies 1-spatiotemporal event privacy with only slight privacy budget reduction, and satisfies 0.5spatiotemporal event privacy with few budget reduction, but

\footnotetext{
${ }^{4}$ https://www.ibm.com/analytics/data-science/prescriptive-analytics/cplexoptimizer
} 
need to reduce more privacy budgets (to be stricter) in order to achieve 0.1-spatiotemporal event privacy. Similar results can be observed in Fig 7(b) and Fig 8 We also observe that the standard deviation is larger for weaker LPPMs since these privacy budgets need to be frequently calibrated. Hence, we can conclude that a stricter PLM for location privacy can protect spatiotemporal event without much calibration, but a more loose PLM may need to reduce its privacy budget significantly for $\epsilon$-spatiotemporal event privacy.

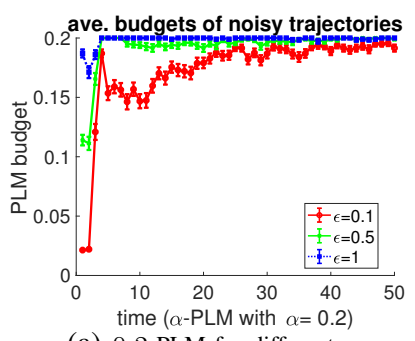

(a) 0.2-PLM for different $\epsilon$.

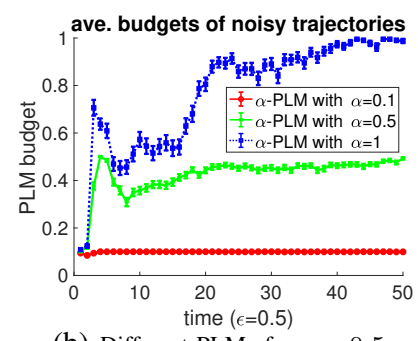

(b) Different PLMs for $\epsilon=0.5$.
Fig. 7: Presence $(\mathbf{S}=\{1: 10\}, \mathbf{T}=\{4: 8\})$ on synthetic data.

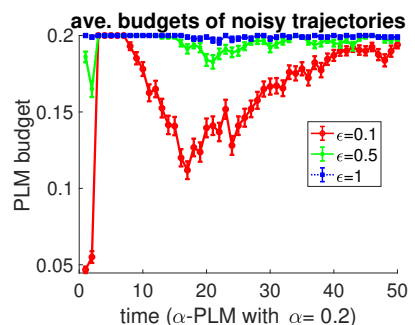

(a) 0.2-PLM for different $\epsilon$.

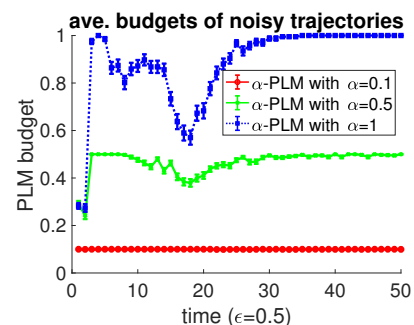

(b) Different PLMs for $\epsilon=0.5$.
Fig. 8: $\operatorname{Presence}(\mathbf{S}=\{1: 10\}, \mathbf{T}=\{16: 20\})$ on synthetic data.

Comparing Fig.7 with Fig 8 , where the events are defined on time periods $4 \sim 8$ and 16 20 respectively, we can see that privacy budgets trend to be reduced during the defined time periods. This indicates that the final $\alpha$ used by PLM at each time point may disclose the definition of spatiotemporal event as we discussion in Section IV-B Hence, a local model is needed for PriSTE framework.

Protecting multiple events. Fig 9 depicts the utilities when protecting two events sequentially using Algorithm 2 . We can see that the utility is much worse than protecting each single event in Fig 7 or Fig 8 because the algorithm needs to simultaneously check if $\epsilon$-spatiotemporal event privacy is satisfied for both events at each time. If no perturbed location satisfying the privacy requirement of both events simultaneously, the algorithm needs to halve the privacy budget until finding an appropriate output.

PriSTE with $\delta$-Location Set Privacy. In Fig 10 , we show the utility of PriSTE with LPPMs that satisfy $\delta$-Location Set Privacy (Algorithm 37. Comparing Fig 10 with Fig 7 . although both of them are using 0.2-PLM, the essential difference between them is the privacy metric: the former satisfies $\delta$-location set privacy and the latter satisfies geoindistinguishability, i.e., 0.2-PLM in Fig 10 has a constrained output domain. We can see that such 0.2-PLM in Fig 10 has to reduce more privacy budgets to achieve $\epsilon$-spatiotemporal event privacy. Intuitively, it is because the privacy metric of $\delta$-location set privacy implies a weaker privacy guarantee due

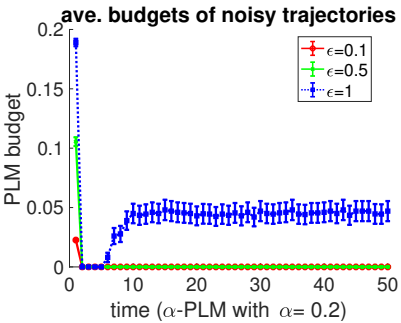

(a) 0.2-PLM for different $\epsilon$

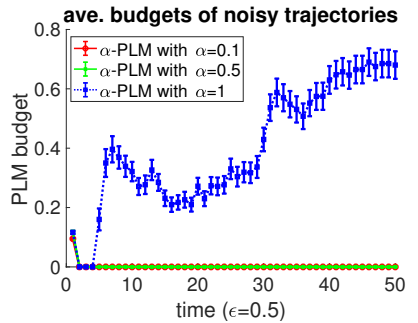

(b) Different PLMs for $\epsilon=0.5$.

Fig. 9: Protecting two events $\operatorname{Presence}(\mathbf{S}=\{1: 10\}, \mathbf{T}=\{4: 8\})$ and Presence $(\mathbf{S}=\{1: 10\}, \mathbf{T}=\{16: 20\})$ on synthetic data.

to the temporal correlations and its LPPM has to be stricter (using a smaller privacy budget) for protecting the event.

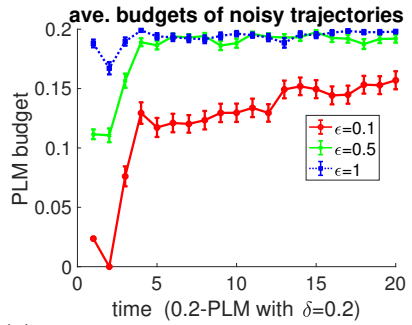

(a) 0.2-PLM with $\delta$-location set privacy for different $\epsilon$.

Fig. 10: $\operatorname{Presence}(\mathbf{S}=\{1: 10\}, \mathbf{T}=\{4: 8\})$ on synthetic data.

\section{Utility over Timestamps}

In this section, we demonstrate the utility against different factors on the Geolife data and synthetic data. Figures 11 and 12 are for protecting PRESENCE event. Due to space limitation, the results of protecting PATTERN event are included in Appendices. Different from the utility in previous section which is averaged at each time, this section displays the utility that is further averaged over time points. Hence, in the left parts of Figures 11 and 12 (ave. budget), the steeper lines indicate the budget may be reduced heavily at some timestamps. Generally, the utility increases with a larger $\epsilon$ in Fig 11 and Fig 12
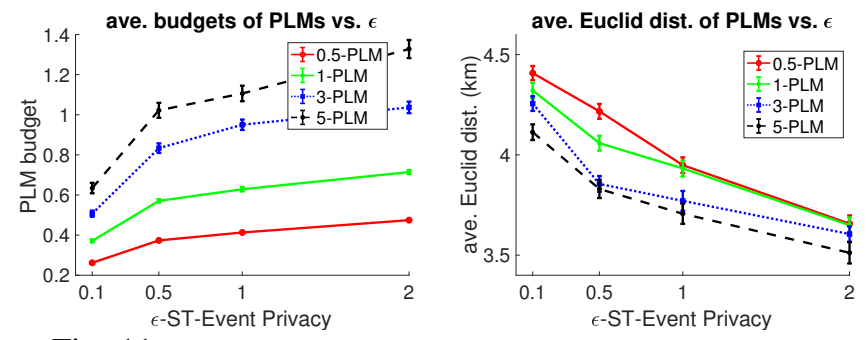

Fig. 11: Presence $(\mathbf{S}=\{1: 10\}, \mathbf{T}=\{4: 8\})$ on Geolife data.

Utility vs. $\alpha$-geo-indistinguishability. In Fig 11 , we can see that a larger $\alpha$-PLM needs to be calibrated heavily (i.e., steeper) for a small $\epsilon$. Interestingly, PLMs who have larger average budgets (in the left figures) may not necessarily have better utility in terms of Euclidean distance. For example, we can see that, at $\epsilon=0.5$, the Euclidean distance of 5-PLM and 3-PLM are very close; at $\epsilon=1$ or 2, 0.5-PLM and 1-PLM appear to have almost the same Euclidean distance. The reason is that PLMs who have larger average budgets may have extremely small budgets at some timestamps, which 
results in the higher average Euclidean distance averaged over time points.
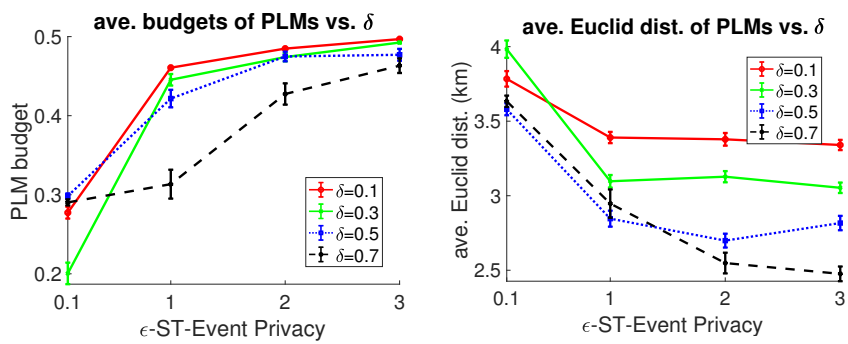

Fig. 12: Presence $(\mathbf{S}=\{1: 10\}, \mathbf{T}=\{4: 8\})$ on Geolife data (0.5-LPM with $\delta$-location set privacy).

Utility vs. $\delta$-location set privacy. In Fig 12 , we can see that a PLM with a larger $\delta$ tends to have a smaller average budget. It is because the PLM with a larger $\delta$ indicates a weaker privacy metric. Hence, the PLM needs to be stricter (i.e, a small budget) to achieve spatiotemporal event privacy. However, such PLM may have a better utility in terms of Euclidean distance as shown in right figure of Fig 12. The reason is that $\delta$-location set privacy with a larger $\delta$ restricts the output domain significantly, which makes perturbed location close to the true location in a high probability. The results are in line with the main purpose of $\delta$-location set privacy: to have a better trade off between utility and privacy.

Utility vs. Transition Matrices. We compare the utility against transition matrices that have different strength of mobility patterns. As we explained previously, a smaller $\sigma$ indicates a more significant mobility pattern. Fig 13 shows that, for the same LPPM, it is hard to protect a spatiotemporal event if user's mobility pattern is significant, i.e., the LPPM needs to be very strict by using a small privacy budget. We also observe that there is no best LPPM for all $\epsilon$-spatiotemporal event privacy in terms of Euclidean distance.
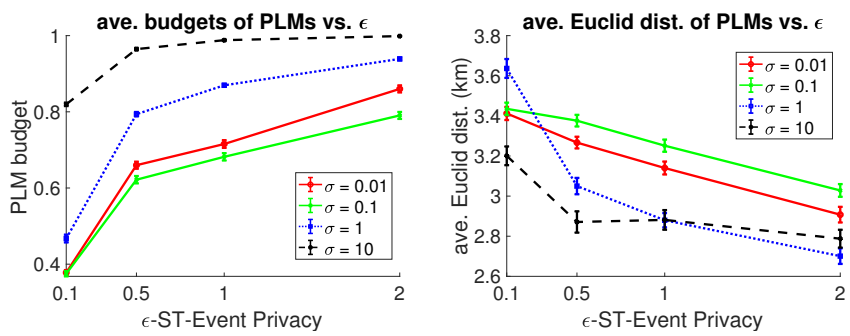

Fig. 13: Presence $(\mathbf{S}=\{1: 10\}, \mathbf{T}=\{4: 8\})$ on synthetic data (1-LPM with geo-indistinguishability).

\section{Runtime}

We name the size of $\mathbf{T}$ and the size of $\mathbf{S}$ as event length and event width, respectively. We also report the performance evaluation on conservative release described in Section IV-B.

Runtime vs. Event Length. We fix the event width as 5 and test 100 random events with length ranging from 5 to 15. Fig 14 shows that the average runtime of the baseline is exponential to event length and the runtime of our method is linear to the event length.

Runtime vs. Event Width. We fix the event length as 5 and test 100 random events with width ranging from 5 to 15 . Fig 14 shows that the average runtime of the baseline is exponential to event width, while our method is polynomial to the event width, which is in line with the complexity of $O\left(\mathrm{~m}^{3}\right)$.
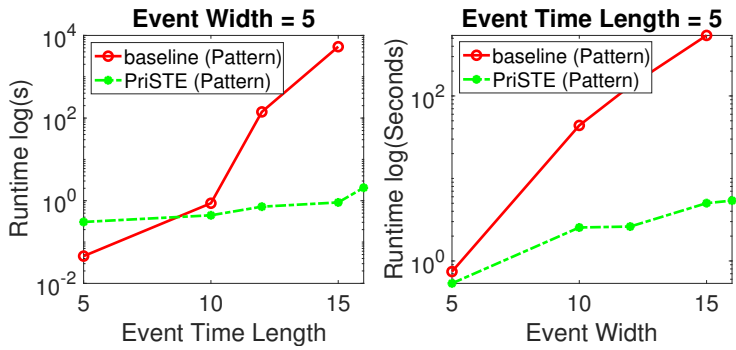

Fig. 14: Runtime

Runtime vs. Conservative Release. In Line 16 of Algorithm 2. we set a threshold runtime in solving the quadratic program. We do not release the perturbed location unless we are sure that Eq. 15 and Eq. 16 are true. The threshold is a trade-off between runtime and utility as shown in Table III among 100 runs. We note that each runtime in Table III includes the whole process of Algorithm 2 In our implementation, we set the threshold to 1 second. We can see as the threshold increases, the number of conservative releases decreases, which results in increasing runtime. On the other hand, the calibrated privacy budgets increasse as the threshold increases. This verifies the tradeoff between runtime and utility that can be achieved by the conservative release.

\begin{tabular}{|l|l|l|l|l|}
\hline $\begin{array}{l}\text { threshold } \\
(\mathbf{s})\end{array}$ & $\begin{array}{l}\text { ave. total } \\
\text { runtime }(\mathbf{s})\end{array}$ & $\begin{array}{l}\text { \# of Con- } \\
\text { Servative } \\
\text { Release }\end{array}$ & $\begin{array}{l}\text { ave. } \\
\text { privacy } \\
\text { budget }\end{array}$ & $\begin{array}{l}\text { ave. } \\
\text { Euclidean } \\
\text { dist. (km) }\end{array}$ \\
\hline 0.01 & 1.1 & $\mathbf{3 3}$ & 0.16 & 2.22 \\
\hline 0.1 & 2.6 & 30 & 0.23 & 1.51 \\
\hline 1 & 5.9 & 21 & 0.22 & 1.52 \\
\hline 2 & 10.4 & 12 & 0.29 & $\mathbf{0 . 9 3}$ \\
\hline 5 & 19.5 & 8 & 0.27 & 1.41 \\
\hline none & $\mathbf{5 2 . 5}$ & 0 & $\mathbf{0 . 3 1}$ & 0.97 \\
\hline
\end{tabular}

TABLE III: Runtime vs. threshold

\section{RELATED WORKS}

\section{A. Location Privacy Preserving Mechanisms}

The LPPMs [8] [9] [20] [21] [22] generally use some obfuscation methods, like spatial cloaking, cell merging, location precision reduction or dummy cells, to manipulate the probability distribution of users' locations. As differential privacy becomes a standard for privacy protection, [8] proposed a Geo-indistinguishability notion based on differential privacy and a planar Laplace mechanism to achieve it. Xiao et al. [9] [18] studied how to protect location privacy under temporal correlations with an optimal differentially private mechanism. Rodriguez-Carrion et al. [23] also studied the effect of temporal dependencies on entropy-based location privacy metric. They proposed a new privacy metric entropy rate and perturbative mechanisms based on it, which can be an alternative LPPM in our framework for protecting spatiotemporal event privacy. Several studies [24] [25] [26] tried to achieve an optimal trade-off between the utility of applications and the privacy guarantee in the LPPMs. Overall, above works all focused on the mechanisms of location privacy, which can be used in our framework as given LPPMs. Whereas we study a new problem of spatiotemporal event privacy. 


\section{B. Inferences on Location}

Various inference attacks can be carried out based on location information and external information such as moving patterns. In the aggregated setting, recent works have studied location or trajectory recovery attacks from aggregated location data [7] [27] or proximity query results from location data [3]. We mainly discuss the individual setting that is closely related to our work. Studies [24] [28] investigated the question of how to formally quantify the privacy of existing LPPMs, given an adversary who can model users' mobility using a Markov process learned from population. [29] used a hierarchical Markov model to learn and infer a user's trajectory based on the places and temporal patterns they visited. [30] used the Continuous Time Bayesian Networks to predict uncertain trajectories of moving objects. Li et al. [31] uses frequent mining approach to find moving objects that move within arbitrary shape of clusters for certain timestamps that are possibly nonconsecutive.

Our privacy check algorithms for computing the prior and posterior probabilities of a spatiotemporal event given perturbed locations are inspired by the work in [32]. Given a Markov process and uncertain locations of moving objects, [32] proposed a modified matrix computation method to efficiently compute the probability of a user appearing in certain region during certain time period. However, the technique in [32] can be only used to compute the probability of the presence of a user in a region during a time period which we refer to as PRESENCE event, whereas we extend the technique to a general set of spatiotemporal events.

\section{CONCLUSION AND Future WORK}

In this paper, we investigate a new type of pivacy goal: protecting spatiotemporal event, which has not been studied in literature. We formally define spatiotemporal events and design a privacy metric extending the notion of differential privacy. We proposed PriSTE, a framework integrating an LPPM for protecting the spatiotemporal event privacy. An interesting direction is to find optimal way for achieving both location privacy and spatiotemporal event privacy. Another question is how can we design a generic mechanism for spatiotemporal event privacy without relying on an LPPM.

\section{REFERENCES}

[1] P. Golle and K. Partridge, "On the anonymity of Home/Work location pairs," in Lecture Notes in Computer Science, 2009, pp. 390-397.

[2] R. Recabarren and B. Carbunar, "What does the crowd say about you? evaluating aggregation-based location privacy," in WPES, vol. 2017, 2017, pp. 156-176.

[3] G. Argyros, T. Petsios, S. Sivakorn, A. D. Keromytis, and J. Polakis, "Evaluating the privacy guarantees of location proximity services," $A C M$ Trans. Priv. Secur., vol. 19, no. 4, pp. 12:1-12:31, 2017.

[4] J. Krumm, "A survey of computational location privacy," Personal and Ubiquitous Computing, vol. 13, no. 6, pp. 391-399, 2009

[5] M. Wernke, P. Skvortsov, F. Dürr, and K. Rothermel, "A classification of location privacy attacks and approaches," Personal and Ubiquitous Computing, vol. 18, no. 1, pp. 163-175, 2014.

[6] K. Chatzikokolakis, E. ElSalamouny, C. Palamidessi, and P. Anna, "Methods for location privacy: A comparative overview," Foundations and Trends® in Privacy and Security, vol. 1, no. 4, pp. 199-257, 2017.
[7] B. Liu, W. Zhou, T. Zhu, L. Gao, and Y. Xiang, "Location privacy and its applications," IEEE Access, pp. 17 606-17 624, 2018.

[8] M. E. Andrés, N. E. Bordenabe, K. Chatzikokolakis, and C. Palamidessi, "Geo-indistinguishability: differential privacy for location-based systems," in CCS, 2013, pp. 901-914.

[9] Y. Xiao and L. Xiong, "Protecting locations with differential privacy under temporal correlations," in CCS, 2015, pp. 1298-1309.

[10] M. Gruteser and D. Grunwald, "Anonymous usage of location-based services through spatial and temporal cloaking," in MobiSys, 2003, pp. $31-42$.

[11] C. Dwork, "Differential privacy: A survey of results," in TAMC, 2008, pp. 1-19.

[12] K. Chatzikokolakis, C. Palamidessi, and M. Stronati, "A predictive differentially-private mechanism for mobility traces," in Lecture Notes in Computer Science, 2014, no. 8555, pp. 21-41.

[13] G. Theodorakopoulos, R. Shokri, C. Troncoso, J.-P. Hubaux, and J.Y. Le Boudec, "Prolonging the hide-and-seek game: Optimal trajectory privacy for location-based services," in WPES, 2014, pp. 73-82.

[14] C. Dwork, M. Naor, T. Pitassi, and G. N. Rothblum, "Differential privacy under continual observation," in STOC, 2010, pp. 715-724.

[15] B. Schuster-Böckler and A. Bateman, "An introduction to hidden markov models," Current Protocols in Bioinformatics, vol. 18, no. 1, pp. A.3A.1-A.3A.9, 2007.

[16] P. M. Pardalos and S. A. Vavasis, "Quadratic programming with one negative eigenvalue is NP-hard," Journal of Global Optimization, vol. 1, no. 1, pp. 15-22, 1991.

[17] C. Dwork, M. Naor, T. Pitassi, G. N. Rothblum, and S. Yekhanin, "Panprivate streaming algorithms." in ICS, 2010, pp. 66-80.

[18] Y. Xiao, L. Xiong, S. Zhang, and Y. Cao, "LocLok: location cloaking with differential privacy via hidden markov model," $V L D B$, vol. 10 no. 12, pp. 1901-1904, 2017.

[19] Y. Zheng, X. Xie, and W.-Y. Ma, "GeoLife: a collaborative social networking service among user, location and trajectory," IEEE Data Eng. Bull., vol. 33, no. 2, pp. 32-39, 2010.

[20] G. Ghinita, M. L. Damiani, C. Silvestri, and E. Bertino, "Preventing velocity-based linkage attacks in location-aware applications," in SIGSPATIAL, 2009, pp. 246-255.

[21] C. A. Ardagna, M. Cremonini, S. D. C. d. Vimercati, and P. Samarati, "An obfuscation-based approach for protecting location privacy," IEEE TDSC, vol. 8, no. 1, pp. 13-27, 2011.

[22] R. H. Hwang, Y. L. Hsueh, and H. W. Chung, "A novel time-obfuscated algorithm for trajectory privacy protection," pp. 126-139, 2014.

[23] A. Rodriguez-Carrion, D. Rebollo-Monedero, J. Forné, C. Campo, C. Garcia-Rubio, J. Parra-Arnau, and S. K. Das, "Entropy-based privacy against profiling of user mobility," Entropy, vol. 17, no. 6, pp. 39133946, 2015.

[24] R. Shokri, G. Theodorakopoulos, J.-Y. Le Boudec, and J.-P. Hubaux, "Quantifying location privacy," in $S P, 2011$, pp. 247-262.

[25] N. E. Bordenabe, K. Chatzikokolakis, and C. Palamidessi, "Optimal geoindistinguishable mechanisms for location privacy," in CCS, 2014, pp. 251-262.

[26] R. Shokri, "Privacy games: Optimal user-centric data obfuscation," PET, vol. 2015, no. 2, 2015.

[27] F. Xu, Z. Tu, Y. Li, P. Zhang, X. Fu, and D. Jin, "Trajectory recovery from ash: User privacy is NOT preserved in aggregated mobility data," in $W W W, 2017$, pp. 1241-1250.

[28] Y. Cao, M. Yoshikawa, Y. Xiao, and L. Xiong, "Quantifying differential privacy under temporal correlations," in ICDE), 2017, pp. 821-832.

[29] L. Liao, D. Fox, and H. Kautz, "Learning and inferring transportation routines," in AAAI, 2004, pp. 348-353.

[30] S. Qiao, C. Tang, H. Jin, T. Long, S. Dai, Y. Ku, and M. Chau, "PutMode: prediction of uncertain trajectories in moving objects databases," Applied Intelligence, vol. 33, no. 3, pp. 370-386, 2010.

[31] Z. Li, B. Ding, J. Han, and R. Kays, "Swarm: Mining relaxed temporal moving object clusters," VLDB, vol. 3, no. 1-2, pp. 723-734, 2010.

[32] T. Emrich, H. P. Kriegel, N. Mamoulis, M. Renz, and A. Zufle, "Querying uncertain spatio-temporal data," in ICDE, 2012, pp. 354-365. 


\section{APPENDIX A}

PROOFS

\section{A. Proof of Lemma III.2}

Proof. At timestamps $1, \boldsymbol{\alpha}_{1}=[\boldsymbol{\pi}, \mathbf{0}] \circ \tilde{\mathbf{p}}_{o_{1}}$. For $t>1, \boldsymbol{\alpha}_{t}=$ $\boldsymbol{\alpha}_{1} \mathbf{M}_{1} \tilde{\mathbf{p}}_{o_{2}}^{\mathbf{D}} \cdots \mathbf{M}_{t-1} \tilde{\mathbf{p}}_{o_{t}}^{\mathbf{D}}=[\boldsymbol{\pi}, \mathbf{0}]\left(\tilde{\mathbf{p}}_{o_{1}}^{\mathbf{D}} \prod_{i=2}^{t}\left(\mathbf{M}_{i-1} \tilde{\mathbf{p}}_{o_{i}}^{\mathbf{D}}\right)\right)$. By Lemma III.1. $\operatorname{Pr}\left(\right.$ Event $\left., o_{1}, o_{2}, \cdots, o_{t}\right)=\boldsymbol{\alpha}_{t} \prod_{i=t}^{\text {end } d-1} \mathbf{M}_{i}[\mathbf{0}, \mathbf{1}]^{\top}$. Then Equation (13) can be derived.

\section{B. Proof of Lemma III.3}

Proof. $\operatorname{Pr}\left(o_{1}, o_{2}, \cdots, o_{t}\right.$, Event is true $)=\sum_{k} \operatorname{Pr}\left(u_{\text {end }}=\right.$ $k, o_{1}, o_{2}, \cdots, o_{t}$, EVENT is true). By forward-backward algorithm, $\operatorname{Pr}\left(u^{t}=s_{k}, o_{1}, o_{2}, \cdots, o_{t}\right)=\alpha_{t}^{k} \beta_{t}^{k}$. Thus we only need to derive the $\boldsymbol{\alpha}_{\text {end }}$ and $\boldsymbol{\beta}_{\text {end }}$ and compute the sum of $\boldsymbol{\alpha}_{\text {end }} \circ \boldsymbol{\beta}_{\text {end }}$ in the world where the Event is true. By Lemma III.2. $\boldsymbol{\alpha}_{\text {end }}=[\boldsymbol{\pi}, \mathbf{0}]\left(\tilde{\mathbf{p}}_{o_{1}}^{\mathbf{D}} \prod_{i=2}^{\text {end }}\left(\mathbf{M}_{i-1} \tilde{\mathbf{p}}_{o_{i}}^{\mathbf{D}}\right)\right)$. By backward algorithm, $\boldsymbol{\beta}_{\text {end }}=[\mathbf{1}, \mathbf{1}] \prod_{i=t-1}^{\text {end }}\left(\tilde{\mathbf{p}}_{o_{i+1}}^{\mathbf{D}} \mathbf{M}_{i}^{\boldsymbol{\top}}\right)$ with $\boldsymbol{\beta}_{t}=[\mathbf{1}, \mathbf{1}]$. Thus $\operatorname{Pr}\left(\right.$ Event $\left., o_{1}, o_{2}, \cdots, o_{t}\right)=\left(\boldsymbol{\alpha}_{\text {end }} \circ \boldsymbol{\beta}_{\text {end }}\right)[\mathbf{0}, \mathbf{1}]^{\top}=\boldsymbol{\alpha}_{\text {end }}\left(\boldsymbol{\beta}_{\text {end }} \circ\right.$ $[\mathbf{0}, \mathbf{1}])^{\top}$, which is equal to Equation (14).

\section{Proof of Theorem IV.1}

Proof Sketch. By Definition II.4, it is equivalent to prove $f_{1}(\boldsymbol{\pi}) \leq 0$ and $f_{2}(\boldsymbol{\pi}) \leq 0$ where $f_{1}(\boldsymbol{\pi})=\frac{\operatorname{Pr}\left(o_{1}, o_{2}, \cdots, o_{T}, \text { EVENT }\right)}{\operatorname{Pr}(\text { EVENT })}-$ $e^{\epsilon \frac{\operatorname{Pr}\left(o_{1}, o_{2}, \cdots, o_{T}, \neg \text { EVENT }\right)}{\operatorname{Pr}(\neg \text { EVENT })}}$ and $f_{2}(\boldsymbol{\pi})=\frac{\operatorname{Pr}\left(o_{1}, o_{2}, \cdots, o_{T}, \mathcal{L} \text { - EVENT }\right)}{\operatorname{Pr}(\neg \text { EVENT })}-$ $e^{\epsilon \frac{\operatorname{Pr}\left(o_{1}, o_{2}, \cdots, o_{T}, \text { EVENT }\right)}{\operatorname{Pr}\left(\mathrm{EVENNT}^{2}\right)}}$. By Lemma III.1 and III.3. Theorem IV.1 can be derived.

\section{APPENDIX B NaIVE Solutions}

\section{A. Computing Prior Probability of an Event}

Considering an event is a set of Boolean expression of (location, time) predicates combined with AND and OR, a naive approach would be to enumerate all possible cases for the event and sum (correspond to OR) the product (correspond to AND) of the probabilities of each location predicate and such an approach would require exponential computation time. Due to space limitation, we omit a detailed algorithm for this naive solution. Instead, we show an example as below.

\section{PATTERN}

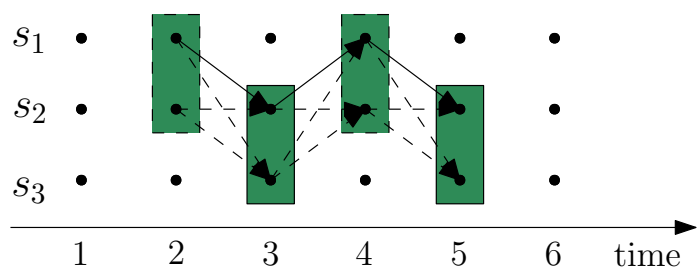

Fig. 15: $\operatorname{Pr}($ PAtTERN $)=\sum \operatorname{Pr}($ all trajectories rendering PATTERN $)$ for the $2^{4}$ trajectories satisfying the PATTERN event defined above.

Example B.1. In Fig 15, a PATTERN event is defined by the shaded regions during timestamp 2 to 5 . Thus start $=2$, end $=5$. The regions are $\boldsymbol{s}_{2}=[1,1,0]^{\top}, \boldsymbol{s}_{3}=[0,1,1]^{\top}$, $\boldsymbol{s}_{4}=[1,1,0]^{\top}, \boldsymbol{s}_{5}=[0,1,1]^{\top}$. To derive the probability of one trajectory, e.g. the solid lines in Fig.15, it would be $\operatorname{Pr}\left(u^{2}=s_{1}\right) * \operatorname{Pr}\left(u^{3}=s_{2} \mid u^{2}=s_{1}\right) * \operatorname{Pr}\left(u^{4}=s_{1} \mid u^{3}=\right.$ $\left.s_{2}, u^{2}=s_{1}\right) * \operatorname{Pr}\left(u^{5}=s_{1} \mid u^{4}=s_{1}, u^{3}=s_{2}, u^{2}=s_{1}\right)$. Because there are $2^{4}$ trajectories for the PATTERN event, the prior probability of PATTERN, i.e., $\operatorname{Pr}(\mathrm{PATTERN})$ is the sum of $2^{4}$ such probabilities.

\section{B. Computing Join Probability of an Event}

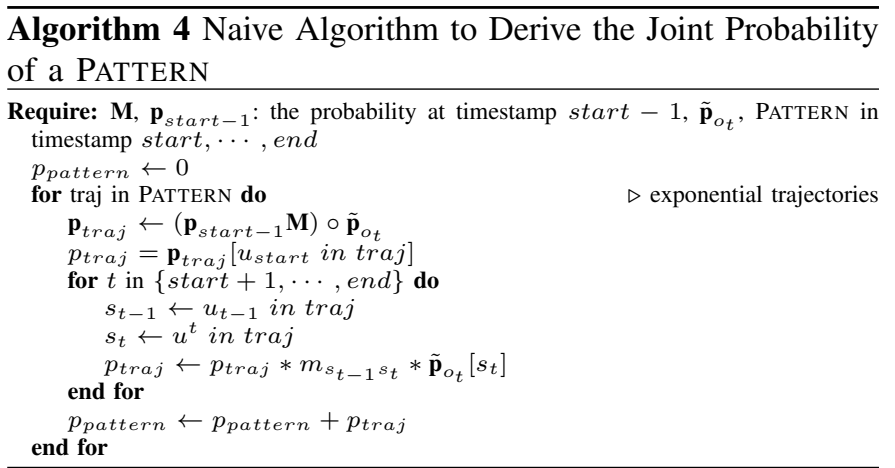

Let traj be a trajectory of the PATTERN. There will be $\mid$ traj $\mid$ trajectories of the PATTERN. For example, in Figure 15 the solid line is traj $=\left\{u^{2}=s_{1}, u^{3}=\right.$ $\left.s_{2}, u^{4}=s_{1}, u^{5}=s_{2}\right\}$, which means $u^{2}$ in traj is $s_{1}$, $\cdots, u^{5}$ in traj is $s_{2}$. Let $\mathbf{M}$ be the transition matrix where $m_{i j}$ is the transition probability from state $i$ to state $j$. $\tilde{\mathbf{p}}_{o_{t}}$ denotes the emission probability of observing $o_{t}$, $\tilde{\mathbf{p}}_{o_{t}}=\left[\operatorname{Pr}\left(o_{t} \mid u^{t}=s_{1}\right), \operatorname{Pr}\left(o_{t} \mid u^{t}=s_{2}\right), \cdots, \operatorname{Pr}\left(o_{t} \mid u^{t}=\right.\right.$ $\left.\left.s_{m}\right)\right]$. Thus $\tilde{\mathbf{p}}_{o_{t}}\left[s_{1}\right]=\operatorname{Pr}\left(o_{t} \mid u^{t}=s_{1}\right), \cdots, \tilde{\mathbf{p}}_{o_{t}}\left[s_{m}\right]=$ $\operatorname{Pr}\left(o_{t} \mid u^{t}=s_{m}\right)$. Let $\operatorname{Pr}\left(o_{\text {start }}, \cdots, o_{\text {end }}\right.$, traj $)$ be the joint probability of the observations and the trajectory. Then $\operatorname{Pr}\left(\right.$ traj $\left.\mid o_{\text {start }}, \cdots, o_{\text {end }}\right)=\frac{\operatorname{Pr}\left(o_{\text {start }}, \cdots, o_{\text {end }}, \text { traj }\right)}{\operatorname{Pr}\left(o_{\text {start }}, \cdots, o_{\text {end }}\right)}$. Thus we focus on the joint probability $\operatorname{Pr}\left(o_{\text {start }}, \cdots, o_{\text {end }}, t r a j\right)$.

Setup. Let start $=2, \mathbf{p}_{\text {start }-1}=\boldsymbol{\pi}$. For a given $\boldsymbol{\pi}$, Algorithm 4 can be used to derive the joint probability of a PATTERN. The runtime can be compared with the runtime of Equation (13), which is also the joint probability.

Note that to derive Equation (13), we prefer "vector*matrix" instead of "matrix*matrix" for efficiency. Calculate it from left to right so that no matrix multiplication should be conducted.

\section{APPENDiX C \\ EXAMPLES}

\section{A. An Example of Prior Probability Computation}

We use an example to describe the details in computation. Example C.1 (Prior Probability Computation). Let us consider the computation of Example III.1. For the PRESENCE event defined at $t=3$ and $t=4$, the transition matrix at $t=2$ and $t=3$ derived by Equation (4) is the left matrix below; while the transition matrix at $t=1$ and $t \geq 4$ derived by Equation (5) is the right matrix below.

$\left.\begin{array}{cccccc}0 & 0 & 0.7 & 0.1 & 0.2 & 0 \\ 0 & 0 & 0.5 & 0.4 & 0.1 & 0 \\ 0 & 0 & 0.9 & 0 & 0.1 & 0 \\ 0 & 0 & 0 & 0.1 & 0.2 & 0.7 \\ 0 & 0 & 0 & 0.4 & 0.1 & 0.5 \\ 0 & 0 & 0 & 0 & 0.1 & 0.9\end{array}\right]\left[\begin{array}{cccccc}0.1 & 0.2 & 0.7 & 0 & 0 & 0 \\ 0.4 & 0.1 & 0.5 & 0 & 0 & 0 \\ 0 & 0.1 & 0.9 & 0 & 0 & 0 \\ 0 & 0 & 0 & 0.1 & 0.2 & 0.7 \\ 0 & 0 & 0 & 0.4 & 0.1 & 0.5 \\ 0 & 0 & 0 & 0 & 0.1 & 0.9\end{array}\right]$

Thus by Lemma III.1. $\operatorname{Pr}(\operatorname{PrESENCE})=[\boldsymbol{\pi}, \boldsymbol{0}] \boldsymbol{M}_{1} \boldsymbol{M}_{2} \boldsymbol{M}_{3}[\boldsymbol{0}, \boldsymbol{1}]^{\top}$, which is a function of $\boldsymbol{\pi}$ as $\operatorname{Pr}(\operatorname{PRESENCE})=\boldsymbol{\pi}[0.28,0.298,0.226]^{\top}$. 